\title{
Mathematical Modelling of the Effects of Mitotic Inhibitors on Avascular Tumour Growth
}

\author{
J. P. WARD* and J. R. KING \\ Department of Theoretical Mechanics, University of Nottingham, Nottingham, NG7, 2RD, UK
}

(Received 29 April 1998; In final form 26 June 1998)

\begin{abstract}
In this paper we build on the mathematical model of Ward and King (1998) to study the effects of high molecular mass mitotic inhibitors released at cell death. The model assumes a continuum of living cells which, depending on the concentration of a generic nutrient, generate movement (described by a velocity field) due to the changes in volumes caused by cell birth and death. The necrotic material is assumed to consist of two diffusible materials: 1) basic cellular material which is used by living cells as raw material for mitosis; 2) a generic non-utilisable material which may inhibit mitosis. Numerical solutions of the resulting system of partial differential equations show all the main features of tumour growth and heterogeneity. Material 2) is found to act in an inhibitive fashion in two ways: i) directly, by reducing the mitotic rate and ii) indirectly, by occupying space, thereby reducing the availability of the basic cellular material. For large time the solutions to the model tend either to a steady-state, reflecting growth saturation, or to a travelling wave, indicating continual linear growth. The steady-state and travelling wave limits of the model are derived and studied, the regions of existence of these two types of long-time solution being explored in parameter space using numerical methods.
\end{abstract}

Keywords: Tumour growth, growth inhibitors. mathematical modelling, numerical solution, asymptotic behaviour

\section{INTRODUCTION}

In the early (avascular) stage of tumour growth, tumour cells acquire nutrient through its diffusion from the host's existing vasculature. Growth during this phase is thought to resemble that of multicell spheroid cultures, an in vitro model of tumour growth (Folkman and Hochberg, 1973). The observed growth pattern of these cultures (typically three growth phases, exponential, linear and growth saturation, see Congar and Ziskin, 1983; Inch et al., 1970; Carlsson, 1977) is mainly dependent on the penetration of nutrient by diffusion from the external medium (Carlsson, 1977): however, other factors are known to be involved, including the production of chemicals that inhibit mitosis (discussed below) and cell shedding (Landry et al., 1982; Weiss, 1978; Ward, 1997 and the subject of a future paper). The chemicals involved in mitotic inhibition fall into two main classes, namely 1) those that affect the

*Corresponding author. 
$\mathrm{pH}$ in the spheroid and 2) large protein molecules, often termed chalones, that somehow interfere with the process of mitosis. In multicell spheroids, any growth factors present are purely endogenous and, in the absence of any vasculature, the capacity to expel undesirable chemicals is limited to diffusion, leading to the accumulation of necrotic and waste products within the spheroid. Substances such as lactic acid, which is produced by the failure of the undernourished cells in the core to complete the respiratory process, lead to the lowering of the overall $\mathrm{pH}$ in the spheroid, with the effect of restricting DNA synthesis so lowering the mitotic rate (Acker et al., 1987; Casciari et al., 1992b; Vaupel, et al., 1981). However, the inhibitors at issue in this paper are the several growth inhibitory proteins originating in the necrotic core or found in the inter-cellular matrix (Freyer, 1988; Freyer et al., 1988; Harel et al., 1984; Iwata et al., 1985; Levine et al., 1984: Sharma and Gehring, 1986 and others: see Iversen, 1991). The inhibitive proteins detailed in Freyer et al. (1988), Harel et al. (1984), Iwata et al. (1985) and Levine et al. (1984) have a molecular mass of $O(10000)$, this being $O(100)$ times that of glucose, and it is this type of inhibitor which will be the focus of study in this paper. Such inhibitor molecules are smaller than a single cell by a factor of $O\left(10^{10}\right)$.

There have been numerous investigations using mathematical models of the role of mitotic inhibitors in tumour growth. Greenspan (1972) incorporated inhibitors into a simple nutrient driven growth model as a mechanism for the formation of quiescent regions. He considered separately the cases of the inhibitor source being the products of necrosis and the waste products of living cells. There have been several subsequent studies that extend the assumptions of Greenspan, although all predict similar qualitative behaviour (Maggelakis and Adam, 1990; Maggelakis, 1993; Byrne and Chaplain, 1996). Glass (1973) and others since (Shymko and Glass, 1976; Adam, 1986; Chaplain and Britton, 1993) simplified the Greenspan model and studied only the inhibitor distribution. This involved the analysis of a secondorder ordinary differential equation with an inhibitor source term representing tumour heterogeneity. This approach allowed the determination of the size of the spheroid at which it becomes fully inhibited and growth ceases (saturation). However, such results contradict experimental observations of a dynamic equilibrium at growth saturation, it being known that the cells near the surface are still dividing (Folkman and Hochberg, 1973; Freyer and Sutherland, 1986; Haji-Karim and Carlsson, 1978). All of these models assume that the diffusion rate of the inhibitor like that of the nutrient, is much faster than the rate of spheroid growth; however, for the larger molecules (molecular mass of $O(10000)$ ) this may not be an accurate simplification. Furthermore, many of these models assume that the inhibitor is continually being produced within the necrotic core, contrasting with the model studied in this paper, where the inhibitor is released only through cell death. A slightly different approach is described in Casciari et al. (1992a), in which a model for the cellular respiratory pathway is coupled with a simple spheroid growth model. This enabled the study of the inhibitive effects caused by the lowering of $\mathrm{pH}$ in the spheroid core due to lactic acid production by hypoxic cells. The model provided reasonable predictions for the distribution of each of the chemicals involved, though it failed to capture the final saturation phase. We note that the saturation phase of spheroid growth is mainly controlled by the mechanisms of necrotic volume loss, which is implicit in the Greenspan based models and is discussed in detail in Ward and King (1998).

In Ward and King (1998) a mathematical model of spheroid growth was presented which is capable of predicting all the main phases of growth and heterogeneity (namely necrotic core/quiescent layer/proliferating rim). Here the quiescent regions result from a time lag in cell death in response to deficient nutrient conditions. This model again assumes nutrient driven growth, but also accounts for the requirement for basic cellular material (DNA, large proteins etc.) originating from the nutrient matrix and necrotic material. In this paper we extend this model by assuming that the cell dissociates into two species of necrotic material: 1) basic cellular material (such as proteins, DNA), considered in Ward and King (1998), which can be utilised by living cells 
for construction of new cells and 2) a material of high molecular mass that is not directly utilisable by the cells and may act as a mitotic inhibitor. The high molecular mass of this second species, as with the basic cellular material, has implications on its diffusive properties (contrasting with previous studies which implicitly assume that the inhibitor is rapidly diffusive). A further difference in the current model is that increasing the inhibitor concentration is taken to lead to a continuous decrease in the mitotic rate, consistent with the findings of Harel et al. (1984), rather than mitosis being 'switched off' when a certain threshold concentration is reached. as in previous studies. We note that the addition of an inhibitive species does not in fact significantly change the qualitative behaviour of the model, but, as will be shown, the presence of even small amounts of inhibitor may significantly affect features such as the final saturation size. The model is formulated in the next section, following a similar course to that of Ward and King (1998), resulting with a complex system of nonlinear partial differential equations to describe spheroid growth and inhibitor action. Throughout the paper simpler cases to the full model will be discussed. In Section 3 the full model is solved numerically, revealing that (in the main) there are two important long-time outcomes to the model, namely solutions which tend towards a steadystate (growth saturation) and those approaching a linear growth rate (travelling waves). These longtime outcomes are studied in greater detail in Sections 4 and 5 where the steady-state and travelling wave limits are derived. The bifurcation between these solutions is established and the distribution in parameter space of the steady-state and travelling wave solutions in explored in Section 6.

\section{THE MATHEMATICAL MODEL}

\subsection{Formulation}

The approach to the modelling follows that of Ward and King (1997 and 1998), based on tumour cells forming a continuum. Local volume changes through birth, death and diffusion of material contribute to movement of the cells within the spheroid, this being described by a velocity field. The birth and death rates are assumed to be governed by the local concentration of a generic nutrient (e.g. oxygen and glucose) and also by the availability of the basic cellular material used to construct new cells. At cell death it is assumed that the cell dissociates into fixed quantities of diffusible necrotic material of two types: 1) basic cellular material (such as proteins, DNA) which can be utilised by living cells for construction of new cells and 2) high molecular mass generic material that may act as an inhibitor. We note that it will be shown that the mere presence of the second type of material has 'inhibitive' effects by reducing the concentration of basic cellular material. The inhibitive material can be viewed as a second generic necrotic species containing a mixture of inhibitive molecules (which reduce the mitotic rate) and material that is not utilised by and has no direct effect on living cells. For the remainder of the paper, however, we shall simply refer to the second necrotic species as the inhibitor. Assuming that the molecular volumes of the (generic) basic cellular material and the (generic) inhibitor are $V_{p}$ and $V_{h}$ respectively, we have

$$
\mu_{p} V_{p}+\mu_{h} V_{h}=V_{D}
$$

where $V_{D}$ is the volume of a dead cell and $\mu_{p}$ and $\mu_{h}$ are dimensionless constants, being the number of cellular and inhibitive molecules released by each drying cell. We note that we have assumed that the amount of cellular material and inhibitor released at cell death is the same whether the cell has died through necrosis or through apoptosis. As in Ward and King (1998), a total volume of $\lambda V_{p}$ of cellular material is required at mitosis, leading to the expression

$$
\begin{aligned}
\begin{array}{c}
\text { net volume } \\
\text { change during } \\
\text { mitosis }
\end{array} & =\begin{array}{c}
\text { volume of } \\
\text { a new cell }
\end{array} \\
& =V_{L}-\begin{array}{c}
\text { volume of cellular } \\
\text { material consumed } \\
\text { during mitosis }
\end{array} \\
& -\lambda V_{p}, \quad \text { (2) }
\end{aligned}
$$

where the constant $V_{L}$ is the live cell volume and the dimensionless constant $\lambda$ is the total number of molecules of cellular material consumed. It is assumed that the living cells have some capacity 
for breaking down the inhibitive material, for which these breakdown products is assumed to consist molecules of negligible volume and, for generality, cellular material. This inhibitor breakdown process leads to volume change described by

$$
\begin{aligned}
\begin{array}{c}
\text { net volume loss } \\
\text { by inhibitor } \\
\text { breakdown }
\end{array} & \begin{array}{c}
\text { volume of } \\
\text { an inhibitor } \\
\text { molecule }
\end{array} \\
= & V_{h}-\begin{array}{c}
\text { cellular material } \\
\text { gained from } \\
\text { breakdown }
\end{array} \\
& -\quad v V_{p}, \quad \text { (3) }
\end{aligned}
$$

where $v$ is a dimensionless constant, representing the number of cellular material molecules produced for every inhibitor molecule broken down, such that $\nu V_{p} \leq V_{h}$ implies an overall volume loss. Here, the molecules of negligible volume produced by such a breakdown process are assumed to diffuse rapidly out of the tumour and do not contribute to its volume. We note the case $v=0$ implies that no cellular material is produced by the breakdown process. Assuming that all space in the tumour is occupied by living cells and cellular and inhibitive material leads to the no void condition

$$
V_{L} n+V_{p} p+V_{h} h=1,
$$

where $n$ is the live cell density and $p$ and $h$ are the concentrations of cellular and inhibitive material. We note that these assumptions generalise those of the model of Ward and King (1998), which corresponds to setting $\mu_{h}=0$ in the current model.

Using the above relations, together with the assumptions given in Ward and King (1998), the following system of equations can be derived

$$
\begin{aligned}
& \frac{\partial n}{\partial t}+\nabla \cdot(v n)=\left(k_{m}(c, p, h)-k_{d}(c)\right) n, \\
& \frac{\partial c}{\partial t}+\nabla \cdot(v c)=D \nabla^{2} c-k(c, p, h) n, \\
& \frac{\partial p}{\partial t}+\nabla \cdot(v p)=D_{p} \nabla^{2} p+\mu_{p} k_{d}(c) n \\
& \quad-\lambda k_{m}(c, p, h) n+v \psi h n, \\
& \frac{\partial h}{\partial t}+\nabla \cdot(v h)=D_{h} \nabla^{2} h+\mu_{h} k_{d}(c) n-\psi h n, \\
& \nabla \cdot v=\left(V_{L}-\lambda V_{p}\right) k_{m}(c, p, h) n-\left(V_{L}-V_{D}\right) k_{d}(c) n \\
& \quad-\left(V_{h}-v V_{p}\right) \psi h n+V_{p} D_{p} \nabla^{2} p+V_{h} D_{h} \nabla^{2} h,
\end{aligned}
$$

where the variables $c$ and $v$ are the nutrient concentration and the velocity field, respectively. These equations have the following interpretation:

Equation (5) states that the rate of change in live cell density is given by the difference in rates of birth $\left(k_{m}(c, p, h)\right)$ and death $\left(k_{d}(c)\right)$, the forms of these rate functions being given below. The divergence term on the left-hand side accounts, in the usual way, for the influence of advective effects $(v \cdot \nabla n)$ and local volume changes $(n \nabla \cdot v)$ on the live cell density.

Equation (6) states that the rate of change of nutrient concentration is governed by the rates of consumption by the living cells, $(k(c, p, h) n)$ and by diffusion, which is assumed to satisfy Fick's Law with a constant diffusion coefficient $D$.

Equation (7) states that the rate of change of cellular material concentration is governed by the rates of release at cell death $\left(\mu_{p} k_{d}(c) n\right)$, production through inhibitor breakdown $(\nu \psi h n)$, consumption during mitosis $\left(\lambda k_{m}(c, p, h) n\right)$ and diffusion (described by Fick's Law with constant diffusion coefficient $D_{p}$ ). The constants $\mu_{p}, \lambda$ and $v$ are those introduced above in the formulating (1), (2) and (3). The non-negative constant $\psi$ governs the rate of cellular conversion of the inhibitor, so that if $\psi=0$ no conversion is occuring.

Equation (8) states that the rate of change of inhibitor concentration is governed by the rates of release at cell death $\left(\mu_{h} k_{d}(c) n\right)$, breakdown by the living cells $(\psi h n)$ and diffusion (again described by Fick's Law with constant diffusion coefficient $D_{p}$ ), (1) and (3) being used in constructing the forcing terms.

Equation (9) can be derived from Equations (4), (5), (7) and (8) and accounts for volume generated through birth and death and from the diffusion of cellular and inhibitive material.

In the remainder of this paper we shall assume spherical symmetry, avoiding the need to include constitutive equations for the velocity field, so that 
Equations (5)-(9) together with suitable boundary and initial conditions form a closed system.

The expression for the mitotic rate function extends that used in Ward and King (1998) to include the effects of the inhibitor. It is assumed that the mitotic rate remains bounded and is monotonic decreasing with the inhibitor concentration and the form for $k_{m}$ adopted is

$$
\begin{aligned}
k_{m}(c, p, h)= & A\left(\frac{c^{m_{1}}}{c_{c}^{m_{1}}+c^{m_{1}}}\right)\left(\frac{p^{m_{3}}}{p_{c}^{m_{3}}+p^{m_{3}}}\right) \\
& \times\left(1-P \frac{h^{m_{4}}}{h_{c}^{m_{4}}+h^{m_{+}}}\right),
\end{aligned}
$$

where $A$ is a positive constant, $c_{c}, p_{c}$ and $h_{c}$ are 'critical' concentrations of nutrient, cellular material and inhibitive material, respectively, $m_{1}, m_{3}$ and $m_{4}$ are positive constants and $P$ is a dimensionless constant, with $0 \leq P \leq 1$. We note that for $P=0$ the presence of the inhibitor does not directly affect mitosis. We further note that if we take $P=1$ and $m_{4} \rightarrow \infty$ (reducing the inhibitor part of (10) to a step function), then if $h>h_{c}$ mitosis is completely inhibited; $h_{c}$ then plays a similar role to the threshold concentration adopted in the assumptions of previous models. The expression for the death rate is the same as that used in Ward and King (1997), namely

$$
k_{d}(c)=B\left(1-\sigma \frac{c^{m_{2}}}{c_{d}^{m_{2}}+c^{m_{2}}}\right),
$$

where $B, \sigma, c_{d}$ and $m_{2}$ are non-negative constants, with $0 \leq \sigma \leq 1$. This form for the death rate function, $k_{d}$, implies cell death occurs even at optimal nutrient levels, reflecting cell loss via apoptosis. Using similar ideas to those of Ward and King (1998) in constructing the consumption rate, the form

$$
\begin{aligned}
k(c, p, h)= & A\left(\frac{c^{m_{1}}}{c_{c}^{m_{1}}+c^{m_{1}}}\right)\left(\beta_{1}+\beta_{2}\left(\frac{p^{m_{3}}}{p_{c}^{m_{3}}+p^{m_{3}}}\right)\right. \\
& \left.\times\left(1-P \frac{h^{m_{4}}}{h_{c}^{m_{4}}+h^{m_{4}}}\right)\right)
\end{aligned}
$$

is used, where $\beta_{1}$ and $\beta_{2}$ are positive constants.
Defining $r=|x|$, we study the above system of equations in a spherical geometry. The initial state is a matter of choice but in the simulations which follow we start with a single cancerous cell (although the continuum model will not then be realistic in the very early stages, it is expected to be acceptable as soon as significant number of cells is present). The external medium is assumed to contain cellular material at concentration $p_{0}$ and, for generality, some inhibitor at concentration $h_{0}$. To model experiments concerning the effects of externally introduced inhibitors on spheroids (for example Freyer et al. (1988)) it would be appropriate to set $h_{0}$ at some non-zero value; in all of the simulations which follow except those illustrated in Figure 13 we take $h_{0}=0$. The initial and boundary conditions are therefore

$$
\begin{array}{ll}
\text { at } t=0 & n=1 / V_{L}, p=0, h=0, S=\left(3 V_{L} / 4 \pi\right)^{1 / 3} \\
\text { at } r=0 & \frac{\partial c}{\partial r}=\frac{\partial p}{\partial r}=\frac{\partial h}{\partial r}=v=0 \\
\text { at } r=S & c=c_{0}, D_{p} \frac{\partial p}{\partial r}=Q_{p}\left(p_{0}-p\right) \\
& D_{h} \frac{\partial h}{\partial r}=Q_{h}\left(h_{0}-h\right), \frac{d S}{d t}=v
\end{array}
$$

where $S(t)$ is the radius of the spheroid and is the coordinate of an unknown moving boundary. Robin type boundary conditions have been imposed for both $p$ and $h$ at $r=S(t)$, whereby the flux of material across the tumour surface is assumed to be proportional to the concentration difference there, with $Q_{p}$ and $Q_{h}$ being non-negative constants. For $Q_{p}>0$ and $Q_{h}>0$ the cellular and inhibitive material is able to escape from the spheroid.

Henceforth, we decouple $p$ from the system of equations using the no void condition (4), giving $p=\left(1-V_{L} n-V_{h} h\right) / V_{p}$, and focus on the equations for live cell density, inhibitor concentration, nutrient concentration and velocity.

\subsection{Non-Dimensionalisation}

Denoting dimensionless quantities with carets, the following rescalings based on the initial conditions 
are made

$$
\begin{gathered}
n=\hat{n} / V_{1}, \quad h=\hat{h} / V_{h}, \quad c=c_{0} \hat{c}, \quad v=r_{0} A \hat{v}, \\
t=\hat{t} / A, \quad r=r_{0} \hat{r}, \quad S=r_{0} \hat{S},
\end{gathered}
$$

where $r_{0}=S(0)=\left(3 V_{L} / 4 \pi\right)^{1 / 3}$. For reasons noted in Ward and King (1997), we can adopt a quasisteady simplification for $c$ resulting in the following non-dimensional system of partial differential equations

$$
\begin{aligned}
& \frac{\partial \hat{n}}{\partial \hat{t}}+\hat{v} \frac{\partial \hat{n}}{\partial \hat{r}}=\hat{n} \frac{\hat{D}_{p}}{\hat{r}^{2}} \frac{\partial}{\partial \hat{r}}\left(\hat{r}^{2} \frac{\partial \hat{n}}{\partial \hat{r}}\right)-\hat{n} \frac{\left(\hat{D}_{h}-\hat{D}_{p}\right)}{\hat{r}^{2}} \frac{\partial}{\partial \hat{r}} \\
& \times\left(\hat{r}^{2} \frac{\partial \hat{h}}{\partial \hat{r}}\right)+\hat{n}(\hat{a}(\hat{c}, 1-\hat{n}-\hat{h}, \hat{h}) \\
& -\hat{n} \hat{b}(\hat{c}, 1-\hat{n}-\hat{h}, \hat{h})) \\
& \frac{1}{\hat{r}^{2}} \frac{\partial}{\partial \hat{r}}\left(\hat{r}^{2} \frac{\partial \hat{c}}{\partial \hat{r}}\right)=\hat{k}(\hat{c}, 1-\hat{n}-\hat{h}, \hat{h}) \hat{n}, \\
& \frac{1}{\hat{r}^{2}} \frac{\partial\left(\hat{r}^{2} \hat{v}\right)}{\partial \hat{r}}=\hat{b}(\hat{c}, 1-\hat{n}-\hat{h}, \hat{h}) \hat{n}-\frac{\hat{D}_{p}}{\hat{r}^{2}} \frac{\partial}{\partial \hat{r}} \\
& \times\left(\hat{r}^{2} \frac{\partial \hat{n}}{\partial \hat{r}}\right)+\frac{\left(\hat{D}_{h}-\hat{D}_{p}\right)}{\hat{r}^{2}} \frac{\partial}{\partial \hat{r}}\left(\hat{r}^{2} \frac{\partial \hat{h}}{\partial \hat{r}}\right), \\
& \frac{\partial \hat{h}}{\partial \hat{t}}+\hat{v} \frac{\partial \hat{h}}{\partial \hat{r}}=\frac{\mathcal{D}(\hat{h})}{\hat{r}^{2}} \frac{\partial}{\partial \hat{r}}\left(\hat{r}^{2} \frac{\partial \hat{h}}{\partial \hat{r}}\right)+\hat{h} \frac{\hat{D} p}{\hat{r}^{2}} \frac{\partial}{\partial \hat{r}}\left(\hat{r}^{2} \frac{\partial \hat{n}}{\partial \hat{r}}\right) \\
& +\hat{n}(\hat{l}(\hat{c}, \hat{h})-\hat{b}(\hat{c}, 1-\hat{n}-\hat{h}, \hat{h}) \hat{h})),
\end{aligned}
$$

where $\hat{D}_{p}=D_{p} / r_{0}^{2} A, \quad \hat{D}_{h}=D_{h} / r_{0}^{2} A$ and $\mathcal{D}(\hat{h})=$ $\hat{D}_{h} \hat{h}\left(\hat{D}_{h}-\hat{D}_{p}\right)$ is non-negative. The dimensionless functions $\hat{a}, \hat{b}$ have the same physical interpretations as in Ward and King (1997), representing net birth and volume production rates respectively, and are given by

$\hat{a}(\hat{c}, 1-\hat{n}-\hat{h}, \hat{h})=\hat{k}_{m}(\hat{c}, 1-\hat{n}-\hat{h}, \hat{h})-\hat{k}_{d}(\hat{c})$,

$\hat{b}(\hat{c}, 1-\hat{n}-\hat{h}, \hat{h})=(1-\hat{\lambda}) \hat{k}_{m}(\hat{c}, 1-\hat{n}-\hat{h}, \hat{h})$

$$
-(1-\delta) \hat{k}_{d}(\hat{c})-(1-\hat{v}) \hat{\psi} h n,
$$

where $\hat{\lambda}=V_{p} \lambda / V_{L}, \delta=V_{D} / V_{L}, \hat{\psi}=\psi / A V_{L}, \hat{v}=$ $V_{p} v / V_{h}$ and the functions $\hat{k}_{m}$ and $\hat{k}_{d}$ are given by

$$
\begin{aligned}
& \hat{k}_{m}(\hat{c}, 1-\hat{n}-\hat{h}, \hat{h})=\left(\frac{\hat{c}^{m_{1}}}{\hat{c}^{m_{2}}-\hat{c}_{c}^{m_{1}}}\right) \\
& \quad \times\left(\frac{(1-\hat{n}-\hat{h})^{m_{3}}}{\hat{p}_{c}^{m_{3}}+(1-\hat{n}-\hat{h})^{m_{3}}}\right)\left(1-P \frac{\hat{h}^{m_{4}}}{\hat{h}_{c^{m_{4}}}+\hat{h}^{m_{4}}}\right), \\
& \hat{k}_{d}(\hat{c})=\frac{B}{A}\left(1-\sigma \frac{\hat{c}^{m_{2}}}{\hat{c}^{m_{2}}+\hat{c}_{d}^{m_{2}}}\right),
\end{aligned}
$$

where $\hat{p}_{c}=V_{p} p_{c}$ and $\hat{h}_{c}=V_{h} h_{c}$. The dimensionless consumption rate, $\hat{k}$, is

$$
\begin{gathered}
\hat{k}(\hat{c}, 1-\hat{n}-\hat{h}, \hat{h})=\left(\frac{\hat{c}^{m_{1}}}{\hat{c}_{c}^{m_{1}}+\hat{c}^{m_{1}}}\right)\left(\hat{\beta}_{1}+\hat{\beta}_{2}\right. \\
\left.\left(\frac{(1-\hat{n}-\hat{h})^{m_{3}}}{\hat{p}_{c}^{m_{3}}+(1-\hat{n}-\hat{h})^{m_{3}}}\right)\left(1-P \frac{\hat{h}^{m_{4}}}{\hat{h}_{c}^{m_{4}}+\hat{h}^{m_{4}}}\right)\right),
\end{gathered}
$$

where $\hat{\beta}_{1}=r_{0}^{2} \beta_{1} A / D V_{L} c_{0}$ and $\hat{\beta}_{2}=r_{0}^{2} \beta_{2} A / D V_{L} c_{0}$ and the dimensionless production rate of the inhibitive species, $\hat{l}$, is defined by

$$
\hat{l}(\hat{c}, \hat{h})=\mu \hat{k}_{d}(\hat{c})=\hat{\psi} \hat{h}
$$

where $\mu=V_{h} \mu_{h} / V_{L}$. We note that the choice of scalings imply that $\mu \leq \delta$ (from (1)) and $\hat{v} \leq 1$ (from (3)).

The full set of dimensionless initial and boundary conditions is

at $\hat{t}=0 \quad \hat{n}=1, \hat{h}=0, \hat{S}=1$,

at $\hat{r}=0 \quad \frac{\partial \hat{h}}{\partial \hat{r}}=0, \frac{\partial \hat{c}}{\partial \hat{r}}=0, \hat{v}=0, \frac{\partial \hat{h}}{\partial \hat{r}}=0$,

at $\hat{r}=\hat{S}(\hat{t}) \quad \hat{D}_{p} \frac{\partial \hat{n}}{\partial \hat{r}}+\hat{D}_{p} \frac{\partial \hat{h}}{\partial \hat{r}}=\hat{Q}_{p}\left(1-\hat{n}-\hat{h}-\hat{p}_{0}\right)$

$$
c=1, \frac{d \hat{S}}{d \hat{t}}=\hat{v}, \hat{D}_{h} \frac{\partial \hat{h}}{\partial \hat{r}}=\hat{Q}_{h}\left(\hat{h}_{0}-\hat{h}\right),
$$

where $\hat{Q}_{p}=Q_{p} r_{0} A, \hat{Q}_{h}=Q_{h} r_{0} A, \hat{p}_{0}=V_{p} p_{0}$ and $\hat{h}_{0}=V_{h} h_{0}$. The boundary condition for $\hat{n}$ at $\hat{r}=\hat{S}$ 
results from the substitution of the no void condition into the Robin condition for $p$.

The system of equations thus consists of two nonlinear reaction-diffusion-convection Equations (13) and (16), a second order differential Equation (14) and a first-order partial differential equation for the velocity (15), defined in the region $0<\hat{r}<\hat{S}(\hat{t})$, the unknown $\hat{S}(\hat{t})$ being a moving boundary coordinate. It will be shown that the degeneracy of the diffusion terms (see (13)) can generate steady-state solutions with $n \equiv 0$ in the core, i.e. having a fully developed necrotic core. However, as with the model of Ward and King (1998), steady-state solutions with $n>0$ throughout the spheroid (i.e. with only a partially developed necrotic core) can also occur and the fully/partially necrotic core bifurcation is discussed in more detail Sections 4-6.

The model has four mechanisms for growth retardation, namely volume loss at cell death, material leakage, consumption of the cellular material and mitotic inhibition. Listed below are various special cases that can arise on 'switching off' individual mechanisms by appropriate choices of parameter values.

Basic Model, i.e. the model of Ward and King (1997). This can be derived by dropping the diffusion terms for the necrotic products $\left(\hat{D}_{p}=\hat{D}_{h}=0\right.$ ), the live cell dependency of cellular material $(\hat{\lambda}=0$, $\hat{p}_{c}=0$ ) and the effects and consumption of the inhibitor $(P=\hat{\psi}=0)$. With the dead cell density defined by $\hat{m}=\hat{p}+\hat{h}$, the dimensionless form of the Basic Models is then recovered. We note that neglecting the diffusion terms requires the removal of the boundary conditions for $h$ and $n$.

Inhibitor-free Model, i.e. the model of Ward and King (1998). This can be derived by setting $\mu=0$, so that none of the second species is produced during necrosis, and by the removal of its external supply by setting either $\hat{Q}_{h}=0$ or $\hat{h}_{0}=0$.

Leakage-inhibitor Model. This is derived by setting $\hat{\lambda}=0$ and $\hat{p}_{c}=0$, so mitosis neither depends on nor consumes cellular material.
Consumption-inhibitor Model. Here we set $\hat{Q}_{p}=$ $\hat{Q}_{h}=0$, thus preventing any escape or influx of material, with $\hat{p}_{c}>0$ and $\hat{\lambda}>0$. Non-trivial solutions can then exist only if $\delta-(1-\hat{\nu}) \mu \leq \hat{\lambda}$, the derivation of this result being described in Section 5 .

Inhibitor-only Model. This is derived by preventing leakage $\left(\hat{Q}_{p}=\hat{Q}_{h}=0\right)$, cellular material consumption $\left(\hat{\lambda}=\hat{p}_{c}=0\right)$ and volume loss by inhibitor conversion $(\hat{v}=1)$. It will be shown in Section 5 that steady-state solutions then exist only in the case $\delta=0$.

The carets on all the dimensionless quantities will be dropped for brevity in the rest of the paper.

\section{NUMERICAL RESULTS}

Many of the effects of the inhibitor on spheroid growth predicted by the model are best illustrated by the long-time solutions, and for this reason only a short survey of the transient behaviour is given here. The numerical procedure for the solution of (13)-(16) subject to (18) is essentially the same as that described in Section 3.1 of Ward and King (1998) and we omit the details of the methods used. The parameter set used as the standard in this section is derived from a combination of experimental values (see Ward and King, 1997, 1998) and best estimates. The parameter values of Ward and King (1998) are again used, i.e.

$$
\begin{gathered}
B / A=0.5, \sigma=0.9, c_{c}=0.1, c_{d}=0.1, m_{1}=1, \\
m_{2}=1, \beta_{1}=0.01, \beta_{2}=0, \delta=1, \lambda=1, \\
D_{p}=300, Q_{p}=10, p_{0}=0.1, p_{c}=0.1, m_{3}=1
\end{gathered}
$$

and the remaining parameters are

$$
\begin{gathered}
\mu=0.1, \psi=1, v=1, P=0.3 \\
D_{h}=300, Q_{h}=10, h_{0}=0, h_{c}=0.1, m_{4}=1 .
\end{gathered}
$$


There is very little relevant data available to establish suitable parameter values for the inhibitor and those given here are for the most part estimates leading to reasonable results; only the diffusion coefficient $D_{h}$ and a value for $P$ could be obtained from the experimental literature. The values chosen imply that inhibitor forms $10 \%$ of the necrotic material produced $(\mu=0.1)$ and can be completely converted by the live cells to make the same volume of usable material $(v=1)$. The value used for $P$ is derived from Figure 2 of Harel et al. (1984), which suggests there is about $30 \%$ mitotic inhibition of 3 T3 mouse fibroblast cultures at saturated levels of the inhibitory factor $\operatorname{IDF}_{\mathrm{N}}$. The inhibitor is taken to have the same diffusive and leakage properties as the cellular material, with no inhibitor being present in the external medium. Based on the power law expressions given in Nugent and Jain (1984), relating molecular masses and the diffusion coefficient, the value chosen for the diffusion coefficient $D_{h}$ represents inhibitive molecules of molecular mass of about 10000 .

Figure 1 shows the growth in time of the spheroid and necrotic radii with the above parameter values. The figure demonstrates that the main features of growth are maintained when the inhibitor is included. Close inspection reveals an initial phase of accelerating growth, soon retarding to an apparent linear growth regime, and eventually retarding further (from about $t=100$ ) to saturate at a size $S \approx 112$. Despite the fairly low level of inhibitor production $(\mu=0.1)$ and fairly weak inhibitory effects on mitosis $(P=0.3)$ the saturation size has dropped sharply from the value $S \approx 168$ which occurs for the uninhibited spheroid (Ward and King, 1998). Inspection of the dashed curve in Figure 1 shows that the necrotic core initially expands faster than the spheroid, consistent with the experimental observations of Groebe and Mueller-Klieser (1996) and Tannock and Kopelyan (1986). Eventually the necrotic core size saturates, resulting in a viable rim (taken to be the region with $n \geq 0$.1) width of about 30 cells for the above parameters.

The 'exponential' and 'linear' phases of growth predicted by the model can be made explicit using the same approach to the asymptotic analysis as that described in Appendix 2 of Ward and King (1998) for the limit $B / A=\varepsilon \rightarrow 0$ with $D_{p}, D_{h}, Q_{p}$, $Q_{h}=O(1 / \varepsilon)$. Exponential growth can be shown by considering the additional limit of $\beta_{1}, \beta_{2} \rightarrow 0$

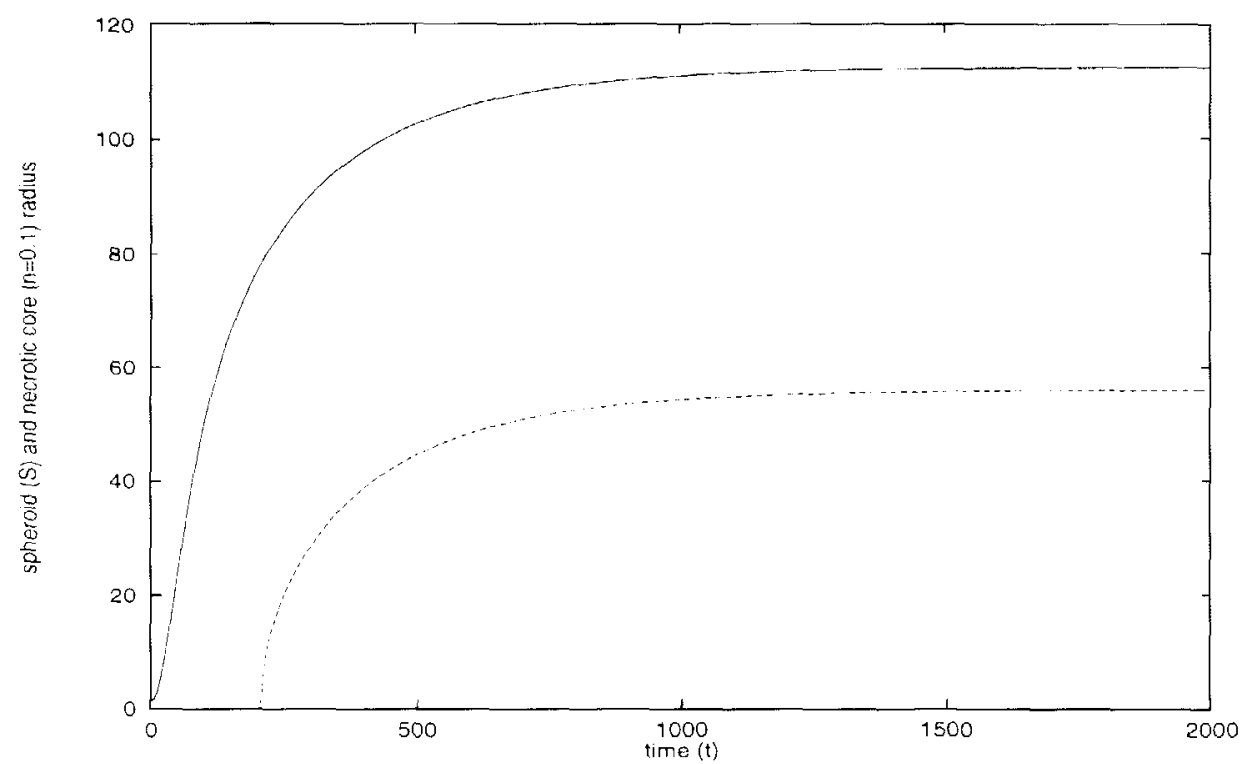

FIGURE 1 Plot of dimensionless tumour radius (solid line) and the necrotic core radius (dashed, defined to be where $n=0.1$ ) against time. 
where we have, following an initial transient, $c \sim 1$, $n \sim 1-p_{0}-h_{0}, h \sim h_{0}$ and

$$
S \sim S_{0} \exp \left(k_{m}\left(1, p_{0}, h_{0}\right) t / 3\right) .
$$

for some positive constant $S_{0}$, provided $t \ll \ln (1 /$ $\left.\left(\beta_{1}+\beta_{2}\right)\right)$. More generally, an equivalent system to Equations (65)-(67) in Appendix 2 of Ward and King (1998) can be derived for the $t=O(1)$ time-scale and, following the initial acceleration of growth, we find that

$$
S \sim \frac{q_{0}\left(m_{1}, c_{c}\right) \eta}{\beta^{1 / 2}} t,
$$

as $t \rightarrow \infty$, where $\eta=\left(p_{0}^{m_{3}} /\left(p_{c}^{m_{3}}+p_{0}^{m_{3}}\right)\right)\left(1-P h_{0}^{m_{4}} /\right.$ $\left.\left(h_{c}^{m_{4}}+h_{0}^{m_{4}}\right)\right), \beta=\left(1-p_{0}-h_{0}\right)\left(\beta_{1}+\beta_{2} \eta\right)$ and $q_{0}$ $\left(m_{1}, c_{c}\right)$ is given by $q=q_{0}\left(m_{1}, c_{c}\right) / \beta^{1 / 2}$, where $q$ is defined in Ward and King (1997). The expression (21) demonstrates that, on the time-scale $t=O(1)$, linear growth is approached in this limit. We note, however, that the expression (21) does not always represent growth in the travelling wave regime discussed in the later sections. Analysis of the longer time-scales (on which growth saturation rather than a travelling wave may occur) leads to a complex system of nonlinear partial differential equations on which limited analytical progress can be made.
The evolution of the live cell density for the simulation of Figure 1 is illustrated in Figure 2. We observe the eventual formation of a plateau of live cells in the viable rim, decreasing deeper into the spheroid to form the necrotic core. The live cell distribution tends to a steady-state with a fully necrotic core, indicated by the solid curve which was obtained from the numerical solution of the appropriate system derived in the next section. The steady-state mitotic rate distribution is given by the dotted curve which demonstrates the existence of a quiescent region of cells towards the edge of the necrotic core. Figure 3 shows the development of the inhibitor distribution in time, about $5.5 \%$ of the material in the necrotic core eventually being inhibitive. The inhibitor profiles are monotonically decreasing in $r$, and are non-zero at the surface, implying that inhibitor leakage is non-negligible and contributes to the volume loss. Even though the concentration of the inhibitor is low, its presence has a significant effect on the overall growth. Using the parameters given above, the maximum inhibitor concentration of about 0.055 will reduce the mitotic rate only by about $10 \%$, which is unlikely on its own to be sufficient to cause such a significant change in saturated spheroid size. The fact that the inhibitor is

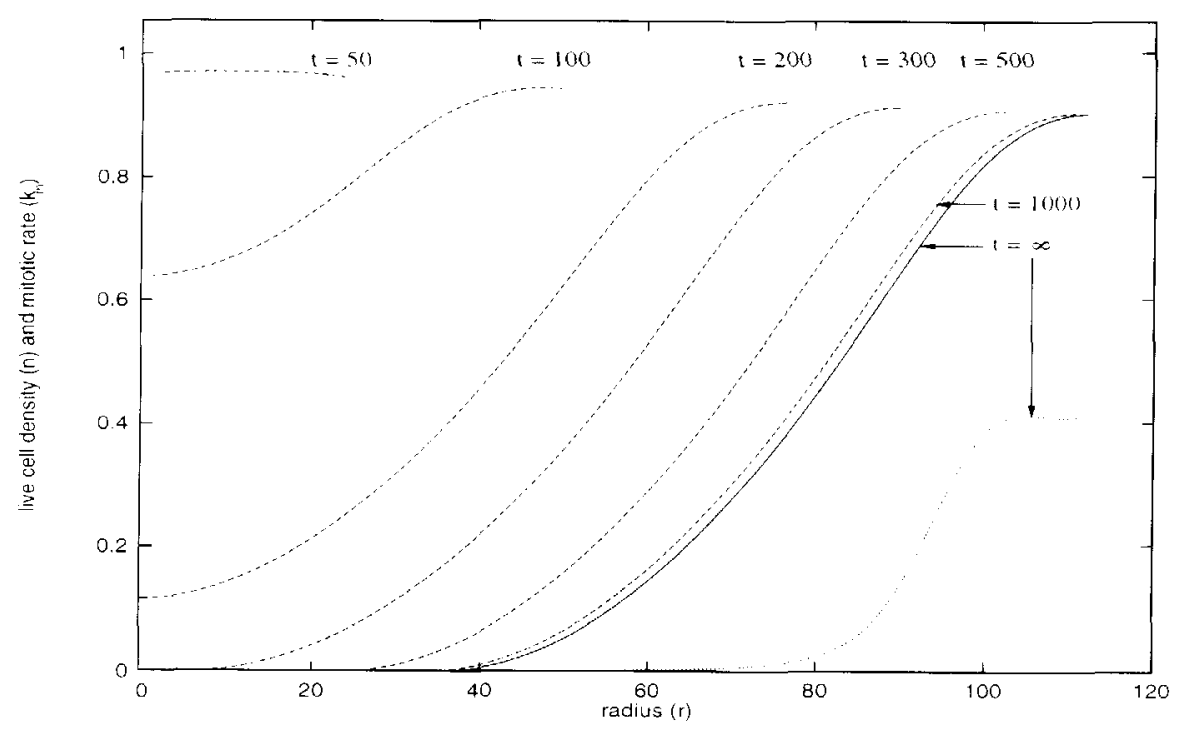

FIGURE 2 Evolution of live cell density distribution in time. The steady-state live cell density and mitotic rate distribution are depicted by the solid and dotted curves respectively. 


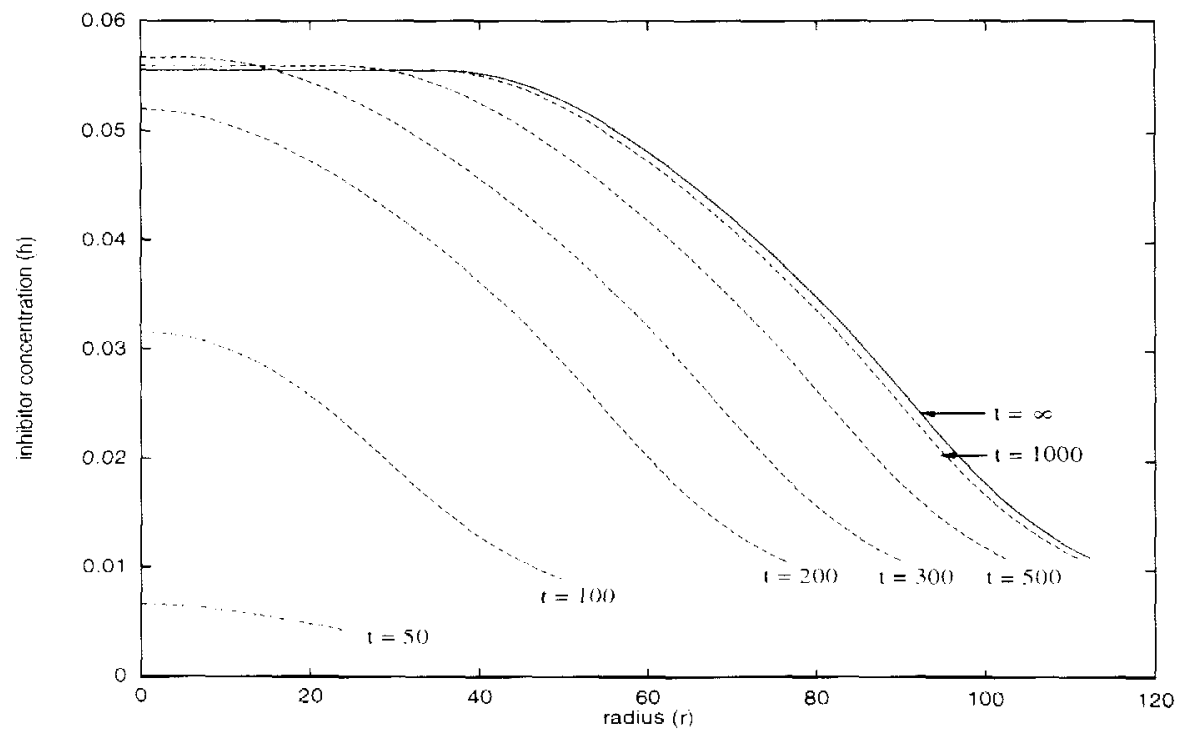

FIGURE 3 Evolution of mitotic inhibitor distribution in time. The solid curve is the steady state solution.

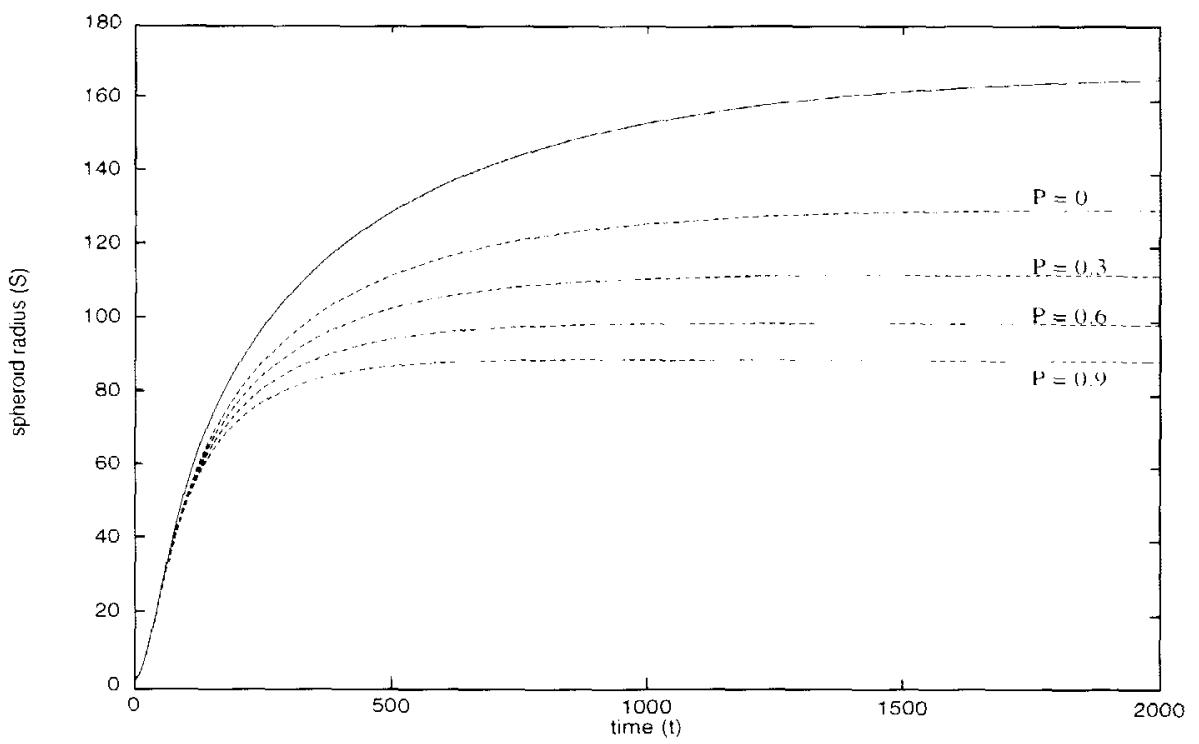

FIGURE 4 Spheroid radius against lime for various values of $P$. The growth of the spheroid without any inhibitor production is depicted by the solid curve, which is taken from Figure 1 of Ward and King (1998).

occupying space that would otherwise be taken up by the cellular material is another important feature. The physical presence of the inhibitor reduces the availability of cellular material and consequently the mitotic rate is reduced.

The role of inhibition by 'space-occupation' is better illustrated in Figure 4, where the effects of the inhibitive strength parameter $P$ are studied, the rest of the parameters being given by (19)-(20). Comparison of the uninhibited growth curve and the $P=0$ curve clearly demonstrates this feature. Here, $P=0$ implies there is no mitotic inhibition and the marked reduction in saturation size (from $S \approx 168$ to $S \approx 130$ ) is due purely to the lowering of cellular 


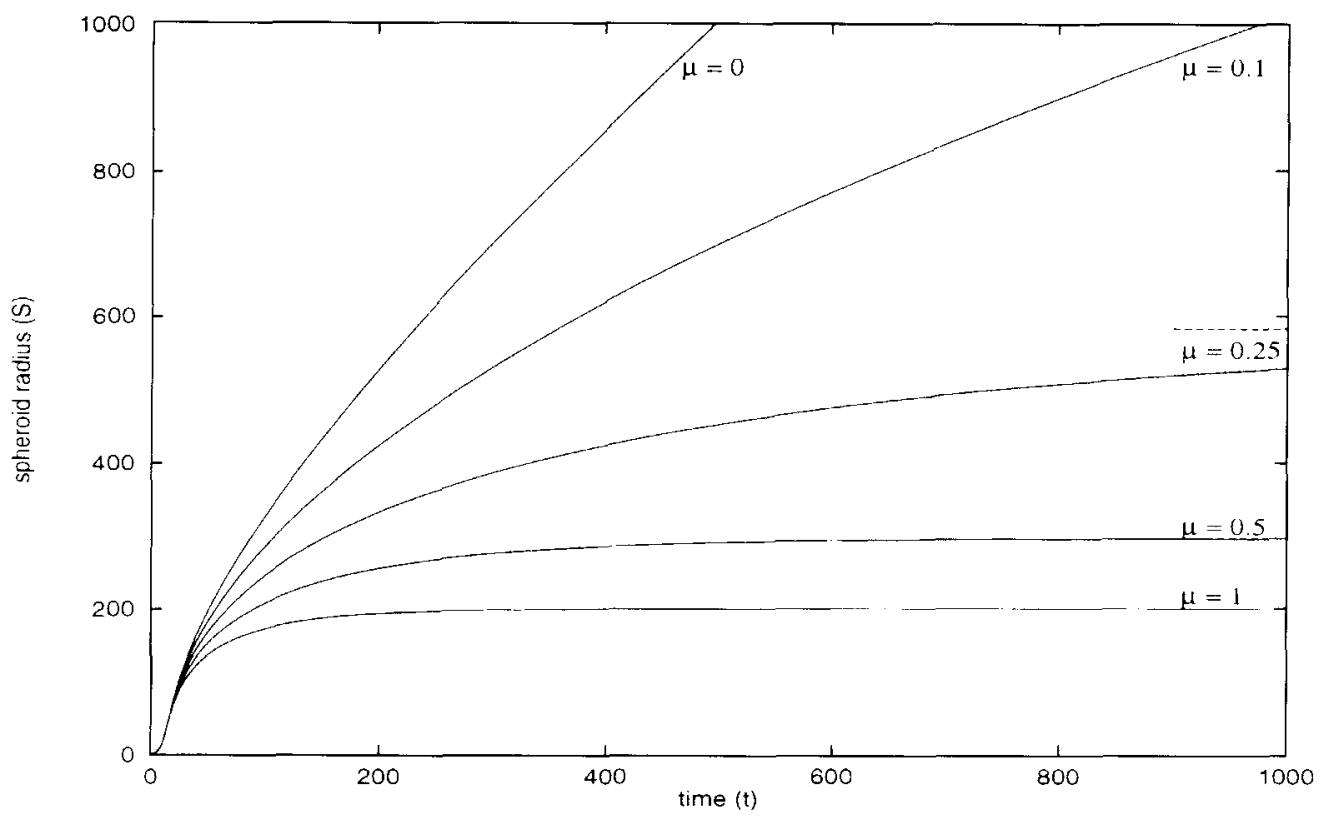

FIGURE 5 Spheroid radius against time for various values of $\mu$ for the Leakage-inhibitor Model. The eventual saturation size is indicated by the dashed lines on the right-hand side.

material availability. This is despite the fact that the inhibitive material can be converted to usable cellular material by the living cells. The figure shows that increasing $P$ has the expected effect of reducing the eventual saturation size. We observe that up to about $t=100$ the curves are indistinguishable, due to the relatively low levels of cell death, and hence of inhibitor production, over this period.

In Figure 5 the effects of the inhibitor production factor $\mu$ on spheroid growth are shown for the Leakage-inhibitor Model. The parameters are given by (19) and (20), except for $D_{p}=D_{h}=800, Q_{p}=$ $Q_{h}=100$ and $\Psi=0$, so that there is no breakdown of inhibitor by the living cells. The curves for $\mu=0$ and $\mu=0.1$ ultimately tend to travelling waves while saturation occurs for $\mu=0.25,0.5$ and 1 ; by solving the large-time equations derived in the next section numerically, the bifurcation between these situations can be shown to occur at $\mu \approx 0.11$. It will be shown in the next section that inhibition as a growth slowing mechanism is inadequate to force growth saturation on its own. However, this figure demonstrates that the amount of inhibitor that is produced may nevertheless be a vital factor in producing growth saturation.

\section{LONG TIME BEHAVIOUR: FORMULATION}

The numerical solutions suggest that, depending on the parameter values, the large-time behaviour is given by either a travelling wave (i.e. continual growth at a linear rate) or a steady-state solution (i.e. growth saturation): as with the Inhibitorfree Model (Ward and King, 1998), the latter may have either a fully or partially developed necrotic core. We note that to our knowledge there is no experimental evidence of the continual growth of spheroids and so that the travelling wave solutions may not be physically relevant. However, their study is necessary to determine the parameter regimes in which continual growth of spheroids is or is not possible; such studies could suggest conditions enabling continued growth to be achieved experimentally. In order to investigate the distribution of these solution types in parameter space, the long time system is now studied. The derivation of the appropriate equations is similar to that of the long time systems for the Inhibitorfree Model. 


\subsection{The Travelling Wave Limit}

The formulation of the relevant system of equations is achieved in the usual manner: we assume the spheroid to be growing at an undetermined constant speed $U>0$, so that $S \sim U t$ as $t \rightarrow \infty$. Translating to the travelling wave coordinates using $z=r-S(t)$, with $z<0$, the following system of ordinary differential equations is obtained

$$
\begin{aligned}
D_{p} n n^{\prime \prime}= & n\left(D_{h}-D_{p}\right) h^{\prime \prime}+(v-U) n^{\prime} \\
& -n(a-b n), \\
c^{\prime \prime}= & k n, \\
v^{\prime}= & b n-D_{p} n^{\prime \prime}+\left(D_{h}-D_{p}\right) h^{\prime \prime}, \\
\mathcal{D}(h) h^{\prime \prime}= & -D_{p} h n^{\prime \prime}+(v-U) h^{\prime}-n(l-b h),
\end{aligned}
$$

where ' denotes $d / d z ; r^{-1} \partial / \partial r$ terms are negligible compared to $\partial^{2} / \partial r^{2}$ terms as $S \rightarrow \infty$. The boundary conditions for this system are

$$
\begin{gathered}
\text { as } z \rightarrow-\infty \quad n^{\prime}, c^{\prime}, v, h^{\prime} \rightarrow 0, \\
\text { at } z=0 \quad D_{p} n^{\prime}+D_{p} h^{\prime}=Q_{p}\left(1-n-h-p_{0}\right), \\
c=1, v=U, \\
D_{h} h^{\prime}=Q_{h}\left(h_{0}-h\right) .
\end{gathered}
$$

The travelling wave system (22)-(25) is seventh order with eight boundary conditions, which are sufficient to determine the seven dependent variables $n, n^{\prime}, c, c^{\prime}, v, h$ and $h^{\prime}$ and the unknown wave speed $U$.

As with model of Ward and King (1998), analysis of the far-field demonstrates that $n$ decays exponentially as $z \rightarrow-\infty$, implying necrosis in the core. In the special cases of 1) $\beta_{1}=0$, so that $k=\beta_{2} k_{m}$ and 2) $\delta=\lambda$, using the same approach described in Ward and King (1998) this condition can be used in reducing the order of the system by one.

\subsection{The Steady-State Limit}

Here the time derivatives are taken to vanish as $t \rightarrow \infty$ and the spheroid to saturate to some undetermined finite radius $S_{\infty}$. The steady-state system consists of the ordinary differential equations

$$
\begin{aligned}
& n \frac{D_{p}}{r^{2}} \frac{d}{d r}\left(r^{2} \frac{d n}{d r}\right)=n \frac{\left(D_{h}-D_{p}\right)}{r^{2}} \frac{d}{d r}\left(r^{2} \frac{d h}{d r}\right) \\
& \quad+v \frac{d n}{d r}-n(a-b n) \\
& \frac{1}{r^{2}} \frac{d}{d r}\left(r^{2} \frac{d c}{d r}\right)=k n \\
& \frac{1}{r^{2}} \frac{d}{d r}\left(r^{2} v\right)=b n-\frac{D_{p}}{r^{2}} \frac{d}{d r}\left(r^{2} \frac{d n}{d r}\right) \\
& \quad+\frac{\left(D_{h}-D_{p}\right)}{r^{2}} \frac{d}{d r}\left(r^{2} \frac{d h}{d r}\right) \\
& \frac{\mathcal{D}(h)}{r^{2}} \frac{d}{d r}\left(r^{2} \frac{d h}{d r}\right)=-h \frac{D_{p}}{r^{2}} \frac{d}{d r}\left(r^{2} \frac{d n}{d r}\right) \\
& \quad+v \frac{d h}{d r}-n(l-b h),
\end{aligned}
$$

defined on the domain $0<r<S_{\infty}$. This system is seventh order with seven dependent variables, $n, d n / d r, c, d c / d r, v, h$ and $d h / d r$ to be determined. The solution can have either a fully or a partially developed necrotic cores and the relevant boundary condition for each case are listed below.

Partially necrotic core solutions: The boundary conditions are

$$
\begin{gathered}
\text { at } r=0 \quad \frac{d n}{d r}=\frac{d c}{d r}=v=\frac{d h}{d r}=0 \\
\text { at } r=S_{\infty} \quad D_{p} \frac{d n}{d r}+D_{p} \frac{d h}{d r}=Q_{p}\left(1-n-h-p_{0}\right), \\
c=1, v=0 \\
D_{h} \frac{d h}{d r}=Q_{h}\left(h_{0}-h\right) .
\end{gathered}
$$

These eight boundary conditions are sufficient to determine the seven dependent variables and the unknown free boundary $S_{\infty}$.

Fully necrotic core solutions: Here there exists another free boundary $R_{\infty}$, where $n$ becomes zero, and for $r<R_{\infty}$ we have $n \equiv 0, v \equiv 0, c=$ $C_{0} \equiv c\left(R_{\infty}\right)$ and $h=H_{0} \equiv h\left(R_{\infty}\right)$, where $C_{0}$ and $H_{0}$ are positive constants. The relevant boundary conditions for the system (27)-(30), defined on 
the region $R_{\infty}<r<S_{\infty}$, are

$$
\begin{gathered}
\text { at } r=R_{\infty} \quad n=\frac{d n}{d r}=\frac{d c}{d r}=v=\frac{d h}{d r}=0 \\
\text { at } r=S_{\infty} D_{p} \frac{d n}{d r}+D_{p} \frac{d h}{d r}=Q_{p}\left(1-n-h-p_{0}\right) \\
c=1, v=0 \\
D_{h} \frac{d h}{d r}=Q_{h}\left(h_{0}-h\right) .
\end{gathered}
$$

For this problem there are now nine boundary conditions to determine the seven variables and the two free boundaries $R_{\infty}$ and $S_{\infty}$.

Fully/partially necrotic core bifurcation: The bifurcation between these two types of solutions occurs when $n(0)=0$ and the boundary conditions are exactly as in (32), with $R_{\infty}=0$. This results in nine boundary conditions to determine the seven variables and the unknown $S_{\infty}$; as expected, the bifurcation problem is thus over-specified since some relation between the parameters must hold in order to lie on the bifurcation curve.

As with the travelling wave case, the order of the system can be reduced by one in the two cases noted above.

\subsection{Travelling Wave/Steady-State Bifurcation}

As with the model of Ward and King (1998), the transition between the two types of large time behaviour corresponds both to vanishing travelling wave speed, $U \rightarrow 0$, and to steady-state spheroid radius tending to infinity, $S_{\infty} \rightarrow \infty$, the two-large time outcomes corresponding to non-intersecting sets of parameters. We locate this bifurcation curve by seeking steady-state solutions with a fully developed necrotic core in the limit of $S_{\infty} \rightarrow \infty$. Focussing on the viable rim region the steady-state Equations $(27)-(30)$ are translated using $x=r-S_{\infty}$, so as $S_{\infty} \rightarrow \infty$ the following system is obtained

$$
\begin{aligned}
& D_{p} n n^{\prime \prime}=n\left(D_{h}-D_{p}\right) h^{\prime \prime}+v n^{\prime}-n(a-b n) \\
& c^{\prime \prime}=k n \\
& v^{\prime}=b n-D_{p} n^{\prime \prime}+\left(D_{h}-D_{p}\right) h^{\prime \prime} \\
& \mathcal{D}(h) h^{\prime \prime}=-D_{p} h n^{\prime \prime}+v h^{\prime}-n(l-b h)
\end{aligned}
$$

where the $r^{-1} d / d r$ terms are of $O\left(S_{\infty}^{-1}\right)$ and are therefore neglected as $S_{\infty} \rightarrow \infty$. Defining the free boundary coordinate $X$ to be the necrotic core interface, so that for $x<X$ we have $n=v=0$, then for $x>X$ we subject Equations (33) $-(36)$ to the boundary conditions

$$
\begin{array}{cc}
\text { at } x=X & n=n^{\prime}=c^{\prime}=v=h^{\prime}=0 \\
\text { at } x=0 & D_{p} n^{\prime}=Q_{p}\left(1-n-h-p_{0}\right)-D_{p} h^{\prime} \\
c=1, v=U \\
D_{h} h^{\prime}=Q_{h}\left(h_{0}-h\right)
\end{array}
$$

Here there are nine boundary conditions to determine the seven variables and the unknown constant $X$; this problem is also over-specified, as is again to be expected, since the parameters must satisfy some relation in order for the solution to lie on the bifurcation curve. We note that the order of the system (33) $-(36)$ can be reduced by one in the special case of $\beta_{1}=0$.

\section{LONG TIME BEHAVIOUR: EXISTENCE OF NON-TRIVIAL SOLUTIONS}

In this section we shall examine the existence of solutions to the long time systems of equations.

\subsection{Existence of Solutions to the Consumption-Inhibitor Model}

Here we examine the existence of non-trivial solutions for the Consumption-inhibitor Model. It will be demonstrated that, except in a very special case, the passage of either cellular or inhibitive material through the surface of the spheroid is essential for the existence of steady-state solutions.

Focussing on travelling wave solutions first, we combine (24) with (22) and (25) to obtain the system

$$
\begin{aligned}
& {[(v-U) n]^{\prime}=\left(k_{m}-k_{d}\right) n,} \\
& v^{\prime}=\left\{(1-\lambda) k_{m}-(1-\delta) k_{d}-\psi(1-v) h\right\} n \\
& \quad-D_{p} n^{\prime \prime}+\left(D_{h}-D_{p}\right) h^{\prime \prime}, \\
& {[(v-U) h]^{\prime}=D_{h} h^{\prime \prime}+\left(\mu k_{d}-\psi h\right) n,}
\end{aligned}
$$


subject to (26); these equations give

$$
\begin{aligned}
v^{\prime}= & \{\delta-\lambda-(1-v) \mu\} k_{m} n+\{1-\delta-(1-v) \mu\} \\
& {[(v-U) n]^{\prime}+(1-v)[(v-U) h]^{\prime}-D_{p} n^{\prime \prime} } \\
& +\left(v D_{h}-D_{p}\right) h^{\prime \prime}
\end{aligned}
$$

Integrating this using (26) and the no flux condition on $z=0$ (recalling that $Q_{p}=Q_{h}=0$ here) we finally obtain

$$
\begin{aligned}
& (1-(1-v) h(-\infty)) U= \\
& \quad(\delta-\lambda-(1-v) \mu) \int_{-\infty}^{0} k_{m} n d z .
\end{aligned}
$$

Unless $v=0$ and $h(-\infty)=1$, positivity of the lefthand side and the non-negativity of the functions $n$ and $k_{m}$ implies the following necessary condition for the existence of travelling wave solutions

$$
\delta-(1-\nu) \mu>\lambda
$$

which is needed to ensure positivity of the righthand side of (39). We note here that at cell death a volume $\delta-\mu$ of cellular material is produced, and a further volume $\nu \mu$ can be gained through conversion of inhibitive material by the living cells. Thus the existence condition (40) simply states that the total amount of cellular material that can be produced through cell death (namely $\delta-(1-\nu) \mu$ ) must exceed that required for birth $(\lambda)$ in order for travelling wave solutions to exist in the case of no material leakage from the spheroid.

Using a similar approach for the steady-state system, (27)-(30)], reveals that non-trivial solutions can only exist if $\delta-(1-v) \mu=\lambda$. As with the Inhibitor-free Model, when $\delta-(1-v) \mu=\lambda$ the zero flux conditions on $n$ and $h$ at $r=S_{\infty}$ imply $v\left(S_{\infty}\right)=0$, so that the system is effectively one boundary condition short: the steady-state system in this case is under-specified and there is an infinite number of solutions parametrised by the saturation size $S_{\infty}$. That the $\delta-(1-v) \mu=\lambda$ case is rather special can also be seen as follows. Using the zero flux conditions for $n$ and $h$ at $r=S(t)$, the timedependent model can be manipulated to give the following expression for $S$

$$
\begin{gathered}
S^{2} \frac{d S}{d t}=\frac{d}{d t} \int_{0}^{S} r^{2}[(1-\lambda) n+(1-v) h] d r \\
\quad-(\lambda-\delta+(1-v) \mu) \int_{0}^{S} r^{2} k_{d l}(c) n d r,
\end{gathered}
$$

which in the special case of $\delta-(1-\nu) \mu=\lambda$, reduces to an exact derivative, namely

$$
\frac{d S^{3}}{d t}=3 \frac{d}{d t} \int_{0}^{S} r^{2}[(1-\lambda) n+(1-v) h] d r
$$

Recalling that $p$ is the concentration of cellular material, so that $p=1-n-h$, this equation may be rewritten to give

$$
\frac{d}{d t} \int_{0}^{s} r^{2}[(\delta-\mu(1-v)) n+p+\nu h] d r=0,
$$

implying that the value of the integral remains fixed for all time. This integral is the total amount of cellular material contained within the spheroid, meaning both the 'free-floating' material $(p)$ and the material that can be produced via cell death $((\delta-$ $\mu(1-v)) n)$ and breakdown of the existing inhibitor $(v h)$. Equation (43) thus states that the total amount of cellular material in the spheroid is conserved during growth as to be expected since $\delta-(1-$ $v) \mu=\lambda$ implies that the total amount of cellular material that can be produced is equal to the amount required for cell birth. Integrating Equation (42) and taking the limit $t \rightarrow \infty$, leads to

$$
\begin{aligned}
& S_{\infty}^{3}-3 \int_{0}^{S_{\infty}} r^{2}[(1-\lambda) n(r, \infty)+(1-v) h(r, \infty)] d r \\
& \quad=S(0)^{3}-3 \int_{0}^{S(0)} r^{2}[(1-\lambda) n(r, 0) \\
& \quad+(1-v) h(r, 0)] d r
\end{aligned}
$$

giving an expression for the steady-state quantities wholly in terms of the initial conditions. Equation (44) provides the extra condition needed for the steady-state case to be a closed system, enabling the saturation size to be determined in terms of the initial conditions. We note that the travelling wave/steady-state bifurcation curve for the Inhibitor-consumption Model is simply the line $\delta-(1-\nu) \mu=\lambda$. 
For $\delta-(1-v) \mu<\lambda$ there are no non-trivial large-time solutions of the Consumption-inhibitor Model; more cellular material is consumed than the maximum amount of cellular material that can be generated by cell death and inhibitive material breakdown, so the tumour must eventually die out.

\subsection{Existence of Solutions of the Inhibitor-Only Model}

For the Inhibitor-only Model we have $\lambda=0$ and $v=1$, and the same analysis leads to $\delta=0$ being the condition for steady-state solutions to exist. Again, there are then an infinite number of solutions parametrised by $S_{\infty}$, dependent on the initial conditions. For $\delta>0$, using a similar argument to that of Section 5.1, it easy to show that travelling wave solutions can exist, but steady-states cannot. The dependence of the long time outcome on $\delta$ is thus similar to that of the Basic Model (Ward and King, 1997); however, in that model the steadystate solution is expected to be unique, as was made explicit in the limit of $B / A \rightarrow 0$, there being sufficient boundary conditions to completely specify the solution.

\subsection{Two Non-Trivial Long Time Solutions}

In Ward and King (1998) it was shown that two non-trivial long time solutions exist in certain parameter regimes, where the bifurcation between the existence of one (Regime I) and two (Regime II) branches of long time solutions was studied by, for example, seeking solutions in the limit of $\lambda \rightarrow \infty$. It was shown that in Regime II the solutions fall on two branches which meet at a finite value of $\lambda$, beyond which no non-trivial long time solutions, of either type, exist; in Regime I, however, a nontrivial long time solution exists for all $\lambda$. Repeating the analysis on the current model in the limit of $\lambda \rightarrow \infty$ we find that $c \sim 1, h \sim h_{0}, n \sim n_{0}$ and

$$
S_{\infty} \sim \frac{Q_{p}}{\lambda} \frac{3\left(n_{0}+p_{0}+h_{0}-1\right)}{n_{0} k_{m}\left(1,1-n_{0}-h_{0}, h_{0}\right)},
$$

where $n_{0}$ is the solution of $k_{m}\left(1,1-n_{0}-h_{0}, h_{0}\right)=$ $k_{d}(1)$, so that

$$
\begin{aligned}
& n_{0}=1-h_{0}-p_{c} \\
& \quad \times\left(\frac{k_{d}(1)\left(1+c_{c}^{m_{1}}\right)\left(h_{c}^{m_{4}}+h_{0}^{m_{4}}\right)}{\left(h_{c}^{m_{4}}+h_{0}^{m_{4}}\right)\left(1-k_{d}(1)\left(1+c_{c}^{m_{1}}\right)\right)-P h_{0}^{m_{4}}}\right)^{\frac{1}{m_{3}}} .
\end{aligned}
$$

Although these limits are not physically realistic, we observe from Equation (45) that positivity of $S_{\infty}$ requires $n_{0}+p_{0}+h_{0}>1$. These expansions for $\lambda \rightarrow \infty$ break down when $n_{0}+p_{0}+h_{0}<1$, indicating that solutions only exist for a finite range of $\lambda$, suggesting Regime II solutions. Thus the line $n_{0}+p_{0}+h_{0}=1$ marks (in the limit) the bifurcation between solution Regimes I and II. The parameters chosen for the numerical work of the next section are such that only Regime I occurs, a single long time solution existing for all $\lambda$.

\section{LONG TIME BEHAVIOUR: NUMERICAL SOLUTIONS}

\subsection{Numerical Methods}

The procedures for the numerical solution of the long time systems follow those of Ward and King (1998). Each of the above cases is reformulated as a two-point boundary value problem and is solved using a shooting and matching method, incorporated in NAG routine D02AGF. The continuation procedure described in Ward and King (1998) is used for studies in parameter space.

\subsubsection{Travelling wave limit}

The linearised solutions of $(22)-(25)$ as $z \rightarrow-\infty$ are used to approximate the variables at a point $z=-L$ for a suitably large value of $L>0$. Defining $\Upsilon=d n / d z, \Psi=d c / d z$ and $\Phi=d h / d z$ and let $y=z / L+1$, we rewrite the $(22)-(25)$ as a system of seven first-order differential equations for $n, \Upsilon, c, \Psi, v, h$ and $\Phi$ to be solved on the region $y \in(0,1)$. Linearising the system $(22)-(25)$ as $z \rightarrow-\infty$ provide approximations to the variables at $z=-L$, leading to the following set of boundary 
conditions

$$
\begin{gathered}
\text { at } y=0 \quad n=N_{0} \exp (a L / U), \\
\Upsilon=-a n / U, c=C_{0}+(U / a)^{2} k n, \\
\Psi=-a\left(c-C_{0}\right) / U, v=-\mathcal{V}_{0} n, \\
h=H_{0}+\mathcal{H}_{0} n, \Psi=-a \mathcal{H}_{0} n / U, \\
\text { at } y=1 \quad \begin{aligned}
n= & N_{1}, \Upsilon=\mathcal{Z}, c=1, v=U, h=H_{1}, \\
\Phi & =Q_{h}\left(h_{0}-H_{0}\right) / D_{h},
\end{aligned}
\end{gathered}
$$

where

$$
\begin{aligned}
\mathcal{Z}= & \frac{Q_{p}}{D_{p}}\left(1-N_{1}-H_{1}-p_{0}\right) \\
& -\frac{Q_{h}}{D_{p}}\left(h_{0}-H_{1}\right) \\
\mathcal{H}_{0}= & -\frac{D_{p} a^{2} H_{0}+U^{2}\left(l-b H_{0}\right)}{a^{2} \mathcal{D}\left(H_{0}\right)-a U^{2}}, \\
\mathcal{V}_{0}= & -\frac{b U}{a}+\frac{D_{p} a}{U}-\left(D_{h}-D_{p}\right) \frac{a}{U} \mathcal{H}_{0},
\end{aligned}
$$

$a$ is given by $a\left(C_{0}, 1-H_{0}, H_{0}\right)$ and similarly for $b, k$ and 1 . Here, the constants $N_{0}, C_{0}, H_{0}, N_{1}$ and $H_{1}$, as well as $U$, are determined as part of the solution; thus, fixing $L$, we have a seventh order system with six unknown parameters and thirteen boundary conditions, and we hence expect the numerical problem to be correctly specified.

\subsubsection{The steady-state limit}

The solution domain is dependent on the type of steady-state solution and we discuss each of these cases separately. The singularities that occur for each of these problems are handled in the same manner as described in Ward and King (1998).

Partially necrotic core solutions: To avoid the evaluation of $r^{-1}$ terms as $r \rightarrow 0$, the boundary conditions are approximated at a point $r=\epsilon$ using a series expansion of the variables in powers of $\epsilon \ll 1$. We again define $\Upsilon=d n / d z, \Psi=a c / d z$ and $\Phi=d h / d z$ and let $y=(r-\epsilon) / L$, where $L=S_{\infty}-\epsilon$, so that $(22)-(25)$ is restated to a system of seven first-order differential equations for $n, \Upsilon, c, \Psi, v, h$ and $\Phi$ to be solved on the region $y \in(0,1)$. The first correction terms of the series expansions are used for the approximation of the boundary conditions at $y=0$, giving

$$
\begin{gathered}
\text { at } y=0 \quad n=N_{0}+\epsilon^{2} \mathcal{N}_{1}, \Upsilon=2 \epsilon \mathcal{N}_{1}, \\
c=C_{0}+\epsilon^{2} k N_{0} / 6, \Psi=\epsilon k N_{0} / 3, \\
v=\epsilon \mathcal{V}_{1}, h=H_{0}+\epsilon^{2} \mathcal{H}_{1}, \Phi=2 \epsilon \mathcal{H}_{1}, \\
\text { at } y=1 \quad n=N_{1}, \Upsilon=\mathcal{Z}, c=1, v=0, \\
h=H_{1}, \Phi=Q_{h}\left(h_{0}-H_{0}\right) / D_{h},
\end{gathered}
$$

where

$$
\begin{aligned}
\mathcal{N}_{1}= & -\frac{1}{6 D_{i} D_{h}}\left(\mathcal{D}\left(H_{0}\right)\left(a-b N_{0}\right)\right. \\
& \left.+\left(D_{h}-D_{p}\right)\left(l-b H_{0}\right) N_{0}\right), \\
\mathcal{H}_{1}= & -\frac{1}{\mathcal{D}\left(H_{0}\right)}\left(D_{p} H_{0} \mathcal{N}_{1}+N_{0} \frac{\left(l-b H_{0}\right)}{6}\right), \\
\mathcal{V}_{1}= & \frac{b}{3} N_{0}-2 D_{p} \mathcal{N}_{1}+2\left(D_{h}-D_{p}\right) \mathcal{H}_{1} .
\end{aligned}
$$

and $\mathcal{Z}$ is given by (46), with $a$ denoting $a\left(C_{0}, 1-\right.$ $\left.N_{0}-H_{0}, H_{0}\right)$ and similarly for $b, k$ and $l$. Thus we have unknown parameters $N_{0}, C_{0}, H_{0}, N_{1}, H_{1}$ and $S_{\infty}$ and for the usual reasons the problem is expected to be well-specified.

Fully necrotic core solutions: The problem of the $1 / n$ term as $r \rightarrow R_{\infty}^{+}$, due to the degeneracy of the 'diffusion' term in Equation (27) is dealt with by solving from a point $r=R_{\infty}+\epsilon$, approximating the variables for $\epsilon \ll 1$. Fixing the domain to the unit interval using $y=\left(r-R_{\infty}-\epsilon\right) / L$, where $L=S_{\infty}-R_{\infty}-\epsilon$, and defining $\Upsilon, \Psi$ and $\Phi$ as before, leads to the same system of equations as for the partially necrotic core case. Using the first correction term of the series expansions for small $\epsilon$ at $y=0$ yields the following set of boundary values

$$
\begin{aligned}
& \text { at } y=0 \quad n=\epsilon^{2} \mathcal{N}_{2}, \Upsilon=2 \epsilon \mathcal{N}_{2} \text {, } \\
& c=C_{0}+\epsilon^{4} k \mathcal{N}_{2} / 12, \Psi=\epsilon^{3} k \mathcal{N}_{2} / 3, \\
& v=\epsilon a / 3, h=H_{0}+\epsilon^{2} \mathcal{H}_{2}, \Phi=2 \epsilon \mathcal{H}_{2}, \\
& \text { at } y=1 \quad n=N_{1}, \Upsilon=\mathcal{Z}, c=1, v=0 \text {, } \\
& h=H_{1}, \Phi=Q_{h}\left(h_{0}-H_{0}\right) / D_{h} .
\end{aligned}
$$


where

$$
\begin{aligned}
& \mathcal{N}_{2}=-\frac{\mathcal{D}\left(H_{0}\right) a}{6 D_{p} D_{h}}, \\
& \mathcal{H}_{2}=\frac{H_{0} a}{6 D_{h}},
\end{aligned}
$$

$a$ denotes $a\left(C_{0}, 1-H_{0}, H_{0}\right)$ and similarly for $k$. We again have the required number unknown constants, namely $C_{0}, H_{0}, N_{1}, H_{1}, S_{\infty}$ and $R_{\infty}$.

Fully/partially necrotic bifurcation: For fixed $\epsilon$ we rescale to the unit interval using $y=(r-\epsilon) / L$ where $L=S_{\infty}-\epsilon$, resulting in the same system as for the partially necrotic core case. To the first correction term, the boundary conditions are for $\epsilon \ll 1$

$$
\begin{aligned}
& \text { at } y=0 \quad n=3 \epsilon^{2} \mathcal{N}_{2} / 5, \Upsilon=6 \epsilon \mathcal{N}_{2} / 5 \text {, } \\
& c=C_{0}+\epsilon^{4} k \mathcal{N}_{2} / 20, \Psi=\epsilon^{3} k \mathcal{N}_{2} / 5, \\
& v=\epsilon a / 5, h=H_{0}+3 \epsilon^{2} \mathcal{H}_{2} / 5, \Phi=6 \epsilon \mathcal{H}_{2} / 5, \\
& \text { at } y=1 \quad n=N_{1}, \Upsilon=\mathcal{Z}, c=1, v=0 \text {, } \\
& h=H_{1}, \Phi=Q_{h}\left(h_{0}-H_{0}\right) / D_{h},
\end{aligned}
$$

where the constants $\mathcal{Z}, \mathcal{N}_{2}$ and $\mathcal{H}_{2}$ are defined above, $a$ denotes $a\left(C_{0}, 1-H_{0} . H_{0}\right)$ and $k$ is defined similarly. Here the quantities to be determined include $C_{0}, H_{0}, N_{1}, H_{1}, S_{x}$ and the relationship which must hold between the parameters in order to lie on the bifurcation.

\subsubsection{Travelling wave/steady-state bifurcation}

To avoid the difficulty with the $1 / n$ term as $x \rightarrow X^{+}$ we integrate from $x=X+\epsilon$ for $\epsilon \ll 1$ and map the system to the unit interval using $y=1+x / L$, where $L=-X-\epsilon$. Defining $\Upsilon, \Psi$ and $\Phi$ as above results in the same system as for the travelling wave case, with $U=0$, together with the boundary conditions (47). The undetermined constants for this case are $C_{0}, H_{0}, N_{1}, H_{1}, X$ and a relationship between the parameters is obtained, locating the bifurcation path.

\subsection{Numerical Results}

The model consists of many parameters and a complete survey of the effects of each of them is impracticable. With little data on any inhibitive species available, the 'standard' set of parameters given below are best guesses. The aim is to assess the qualitative effects of the various parameters and, as in Ward and King (1998), we shall focus mainly on the paths of the travelling wave/steady-state bifurcation in parameter space. We will be restricting attention to solutions under the Regime I parameter scheme (discussed in Section 5.3). The behaviour of the Regime II solutions are not significantly different to that of the uninhibited case of the model, which is studied in more detail in Ward and Kind (1998).

In the following set of figures we again use (19), except that (see below) we take $D_{p}=Q_{p}$, and choose $D_{p}=Q_{p}=100$; the 'standard' set of parameters for the inhibitive species is

$$
\begin{aligned}
& D_{h}=400, \quad Q_{h}=400, \quad \mu=0.1, \quad \Psi=1, \quad v=1, \\
& P=0.9, \quad h_{0}=0, \quad h_{c}=0.1, \quad m_{4}=1 . \quad(48)
\end{aligned}
$$

Throughout we shall set $D_{p}=Q_{p}$ and $D_{h}=Q_{h}$, ensuring that the terms in the Robin boundary conditions for $n$ and $h$ remain balanced, and changes in leakage properties that would result from changing $D_{i} / Q_{i}$, do not obscure the effects of the other parameters. The choice $P=0.9$, rather than $P=$ 0.3 (used in the numerical solutions of the transient model), is made to emphasise the role of the inhibitor in the long time behaviour of the model.

In Figure 6 the locations of travelling waves and of both types of steady-state solution are shown in $\left(D_{h}=Q_{h}, \mu\right)$ space, with the other parameters given above. The solid and dashed curves mark the bifurcations between travelling wave/steady-state and fully/partially necrotic core solutions, respectively. Underneath the solid curve there is insufficient inhibitor production, together with insufficient leakage of necrotic material, for growth saturation to result. In fact, for this example if $D_{h}=$ $Q_{h} \leq 30.69$ then saturation is not possible over the physical range of $\mu$, namely $0 \leq \mu \leq 1$. The numerics suggest that both of the bifurcation curves 


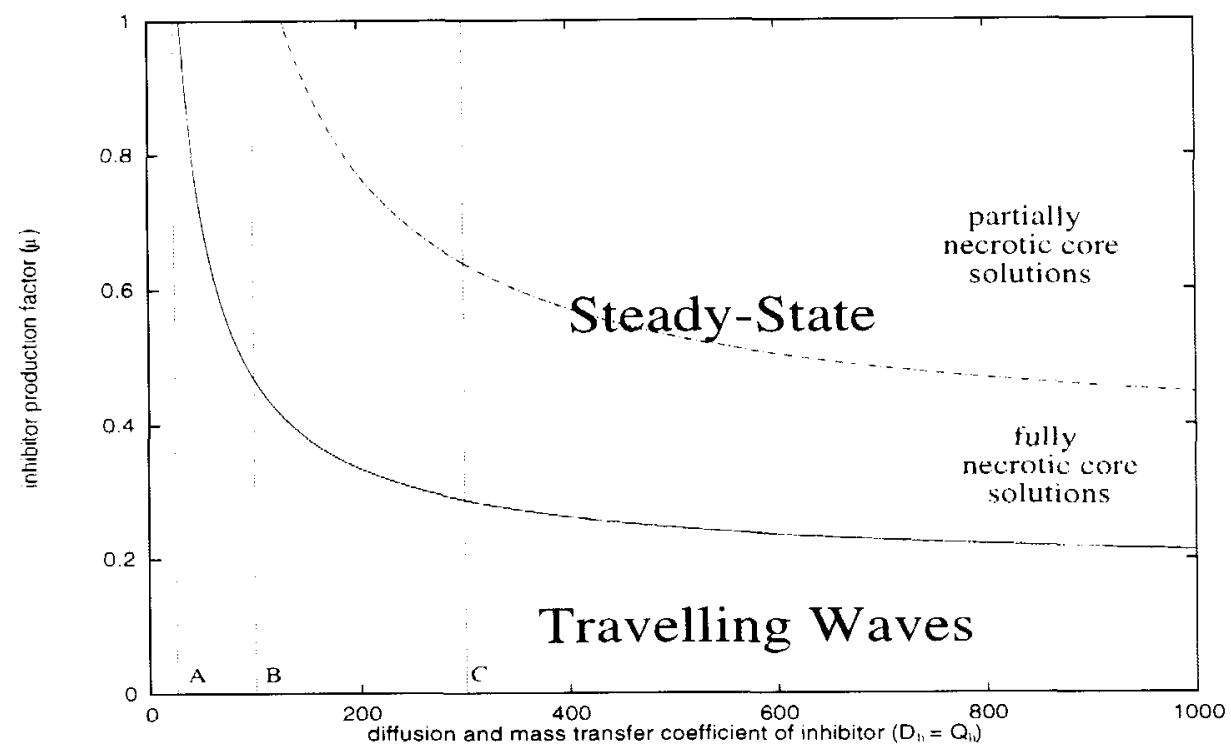

FIGURE 6 The distribution of steady-state and travelling wave solutions in $\left(D_{h}=Q_{h}, \mu\right)$ parameter space, showing the solutions to the travelling wave/steady-state (solid curve) and fully/partially necrotic core (dashed curve) bifurcation formulations. The paths for Figures 7 and 8 are indicated by the dotled lines labelled $\mathbf{A}-\mathbf{C}$; we note that lines $\mathbf{A}$ and $\mathbf{B}$ are not asymptotes of the bifurcation curves.

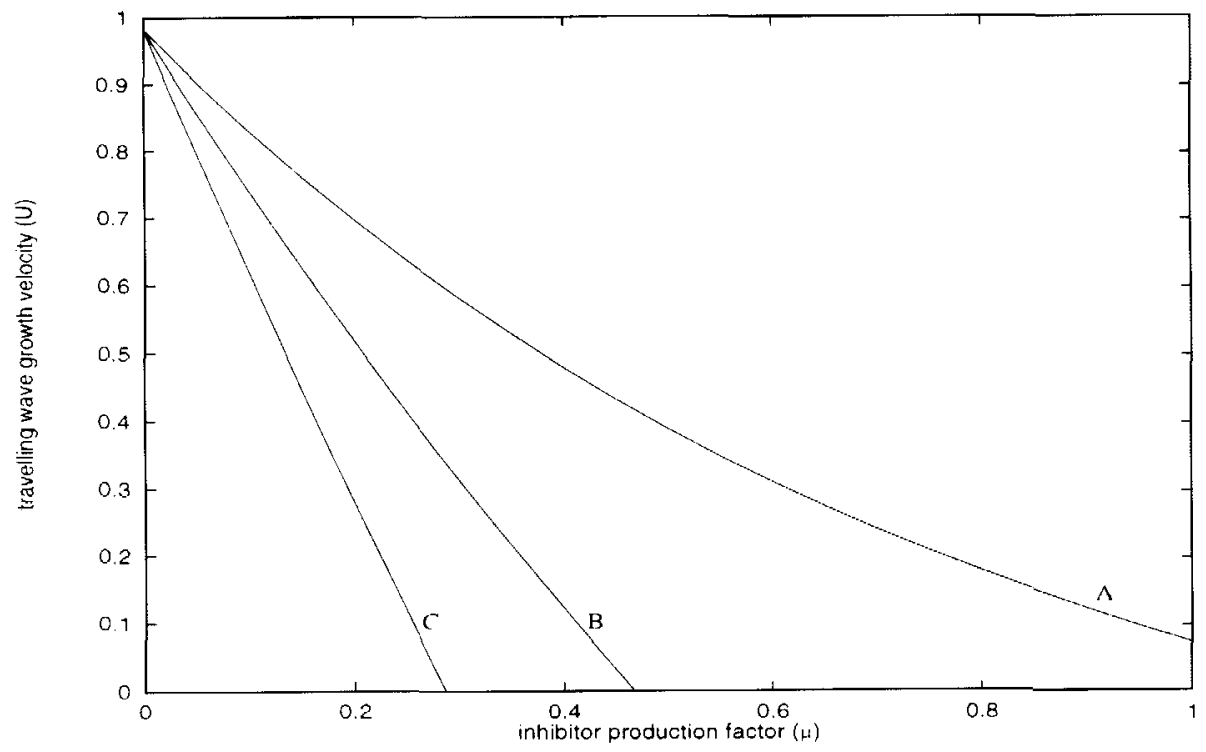

FIGURE 7 Plots of the travelling wave growth speed against $\mu$ for fixed values of $D_{h}=Q_{h}$ equalling 25 (A), $100(\mathbf{B})$ and $300(\mathbf{C})$.

asymptote to a non-zero limit as $D_{h}=Q_{h} \rightarrow \infty$. This is to be expected - as the diffusion of the inhibitor becomes more rapid, less accumulates in the spheroid and the model effectively reduces to the Inhibitor-free Model with a modified cell death contraction factor, $\tilde{\delta}$, given by $\tilde{\delta}=\delta-\mu$.
The dotted lines labelled $\mathbf{A}-\mathbf{C}$ are paths along which the travelling wave speed and saturation size have been investigated as functions of $\mu$; we note that path A lies entirely in the travelling wave region. In Figure 7 we observe that the travelling wave speed is monotonically decreasing in $\mu$, which 
is to be expected as an increase in inhibitor production in the core leads to reduced growth in the viable rim. The expected behaviour that increasing the inhibitor production leads to the decrease in saturation size is demonstrated in Figure 8. The travelling wave/steady-state bifurcations are indicated by the dotted lines, and we see that the saturation size initially decreases rapidly on increasing $\mu$ before levelling off. The dashed curves indicate the radius of the necrotic core, defined to be the position of the necrotic interface; inspection of the figure shows that the viable rim increases in width as more inhibitor is produced. This is due to the increase of inhibitor in the viable rim lowering the live cell density, allowing greater penetration of adequate nutrients; we note $\beta_{2}=0$ implies that nutrient consumption is independent of the inhibitor concentration.

By suitable choice of parameters, the effects of leakage and inhibitor action can be sufficient to preclude the possibility of travelling wave solutions in $\left(D_{h}=Q_{h}, \mu\right)$ space. The results of such a case are illustrated in Figure 9 where we have used the parameter set (19) and (48), except that we have set $D_{p}=Q_{p}=300$. This enables greater leakage of the cellular material, sufficient to ensure that only steadystate solutions exist throughout this parameter space.

In Figures 10-12 we investigate the effects of the inhibitor parameters $\mu, D_{h}=Q_{h}$ and $\psi$ on the travelling wave/steady-state bifurcation curves in $\left(D_{p}=\right.$ $Q_{p}, \lambda$ ) space. All other parameters are given by (19) and (48), except that we set $\mu=0.25$. We note that the travelling wave solutions lie below each of the bifurcation curves shown, with steady-state solution lying in the remainder of the quarter space. We note in Figure 10 that setting $\mu=0$ reduces the current model to the Inhibitor-free Model, the corresponding curve being the same as the solid curve of Figure 12 of Ward and King (1998). Increasing $\mu$ leads to a greater concentration of inhibitor in the viable rim region, reducing the growth rate and, as shown in the figure, resulting in the shrinkage of the travelling wave region. It is worth noting that the bifurcation curves retain a very similar shape for all $\mu$. Similar qualitative behaviour can be observed by varying $v$ across the physical range of $v$, i.e. $0 \leq v \leq 1$, it being found that increasing $v$ leads to the shrinkage of travelling wave region due to more inhibitor being converted and then consumed by the living cells.

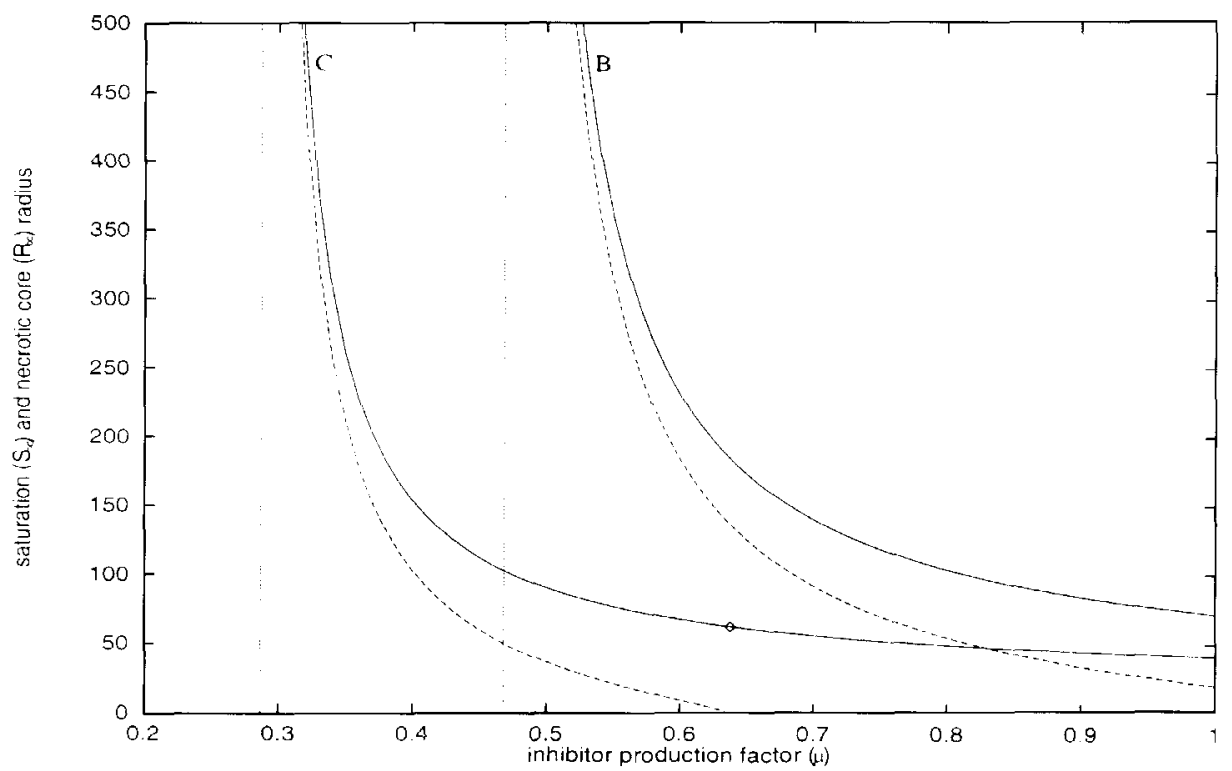

FIGURE 8 Plots of salurated spheroid size (solid) and necrotic core radius (dashed; fully necrotic core interface, $R_{\infty}$ ) against $\mu$ for fixed $D_{h}=Q_{h}$ equalling $100(\mathbf{B})$ and 300 (C). The travelling wave/steady-state and fully/partially necrotic core bifurcations (C only) are indicated by the dotted lines and the ' $\circ$, respectively. The travelling wave solutions lie to the left of the dotted lines. 


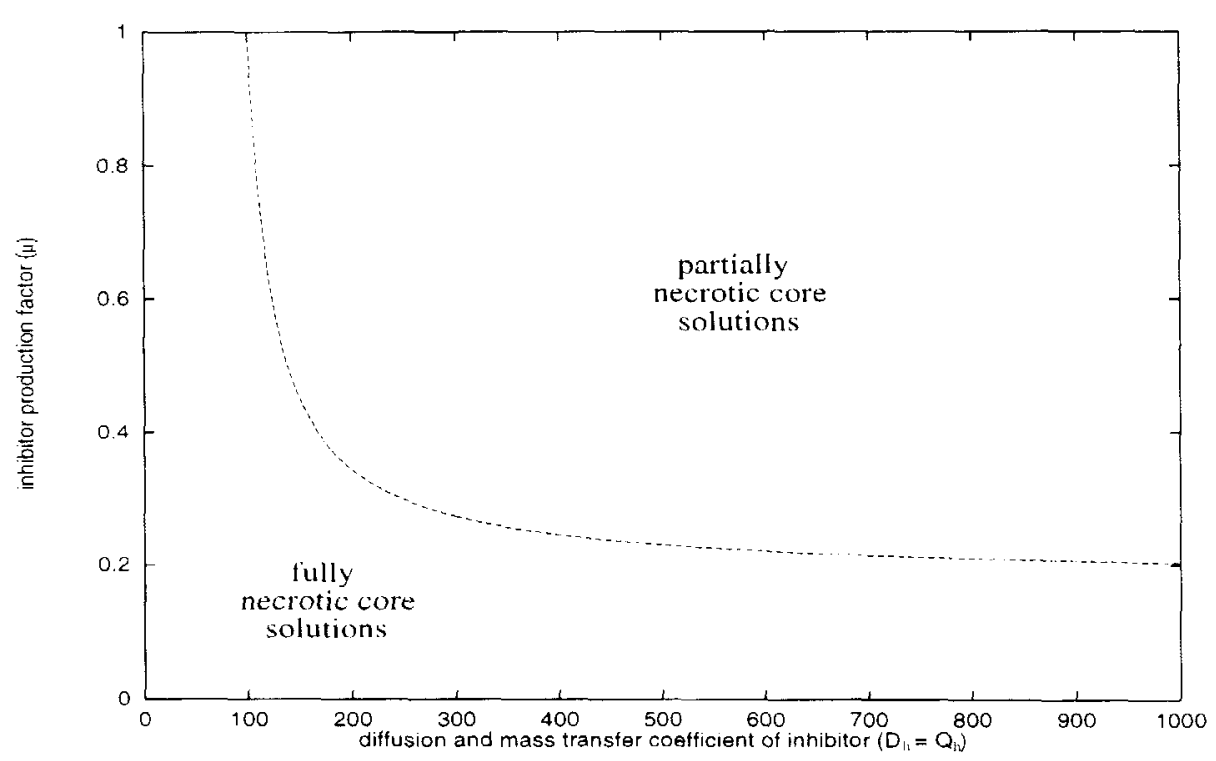

FIGURE 9 The distribution of the two types of steady-state solutions in $\left(D_{h}=Q_{h}, \mu\right)$ space. Parameters given by (48) except for $D_{p}=Q_{p}=300$.

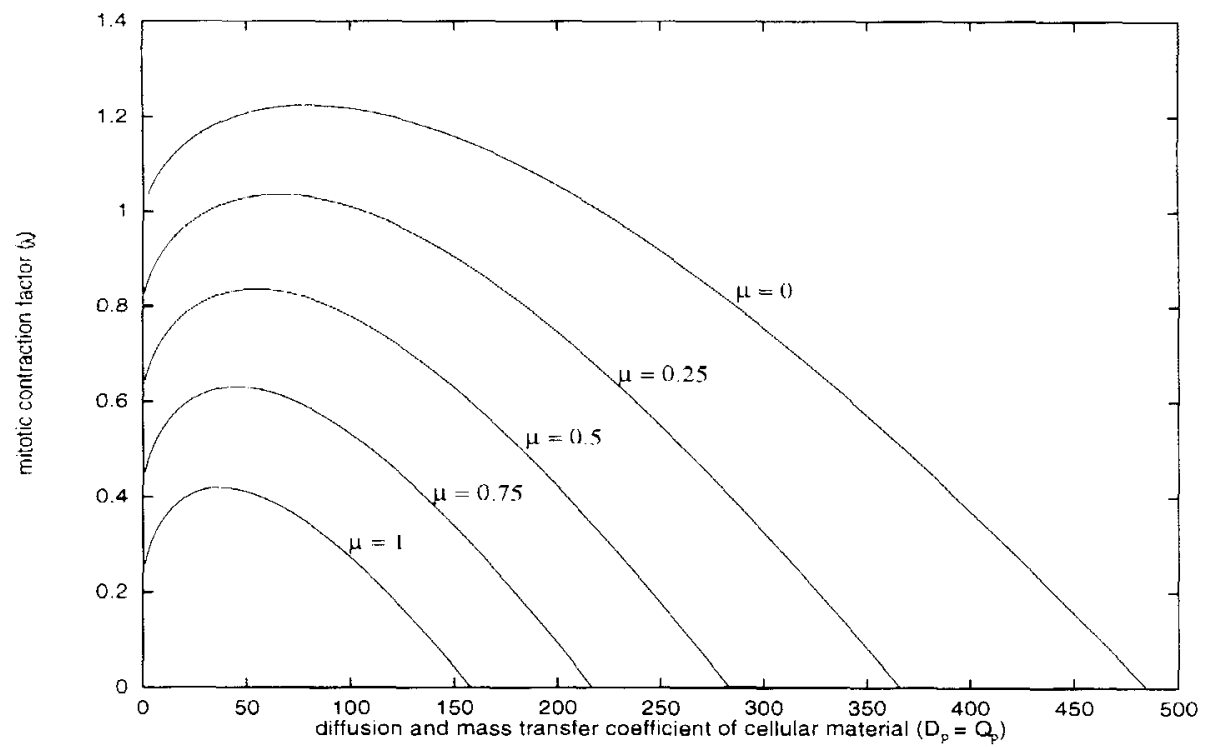

FIGURE 10 The effects of the inhibitor production factor $\mu$ on the travelling wave/steady-state bifurcation in $\left(D_{p}=Q_{p}, \lambda\right)$ space. The travelling wave solutions lie below the bifurcation curves in each of the Figures $10-12$.

Rather different behaviour of the bifurcation curves as a result of varying $D_{h}=Q_{h}$ is shown in Figure 11. The curves for $D_{h}=Q_{h}=40$ and $D_{h}=Q_{h}=400$ are qualitatively similar and the shrinkage of the travelling wave region is due to the increase of the diffusion rate, providing greater penetration of inhibitor into the viable rim from where it may also leak. However, as $D_{h}=Q_{h}$ is increased to 4000 


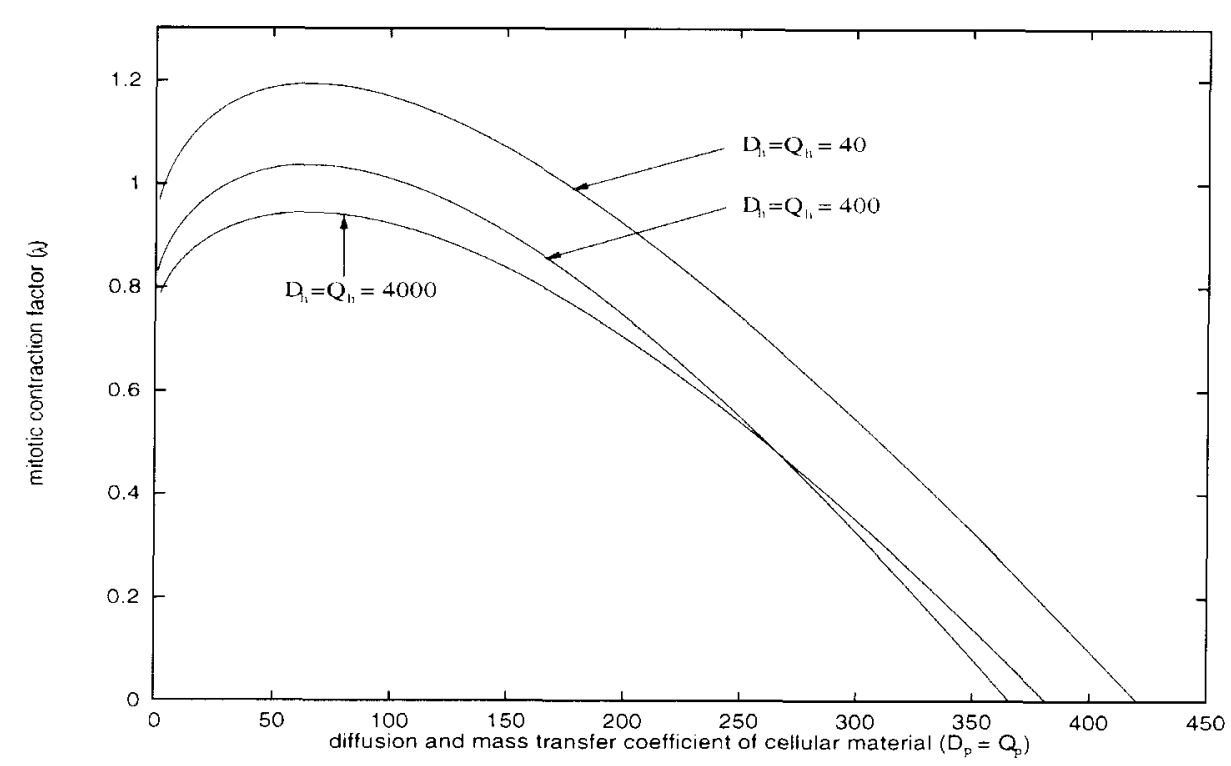

FIGURE 11 The effects of the inhibitor diffusion and mass transfer coefficients $D_{h}=Q_{h}$ on the travelling wave/steady-state bifurcation in $\left(D_{p}=Q_{p}, \lambda\right)$ space.

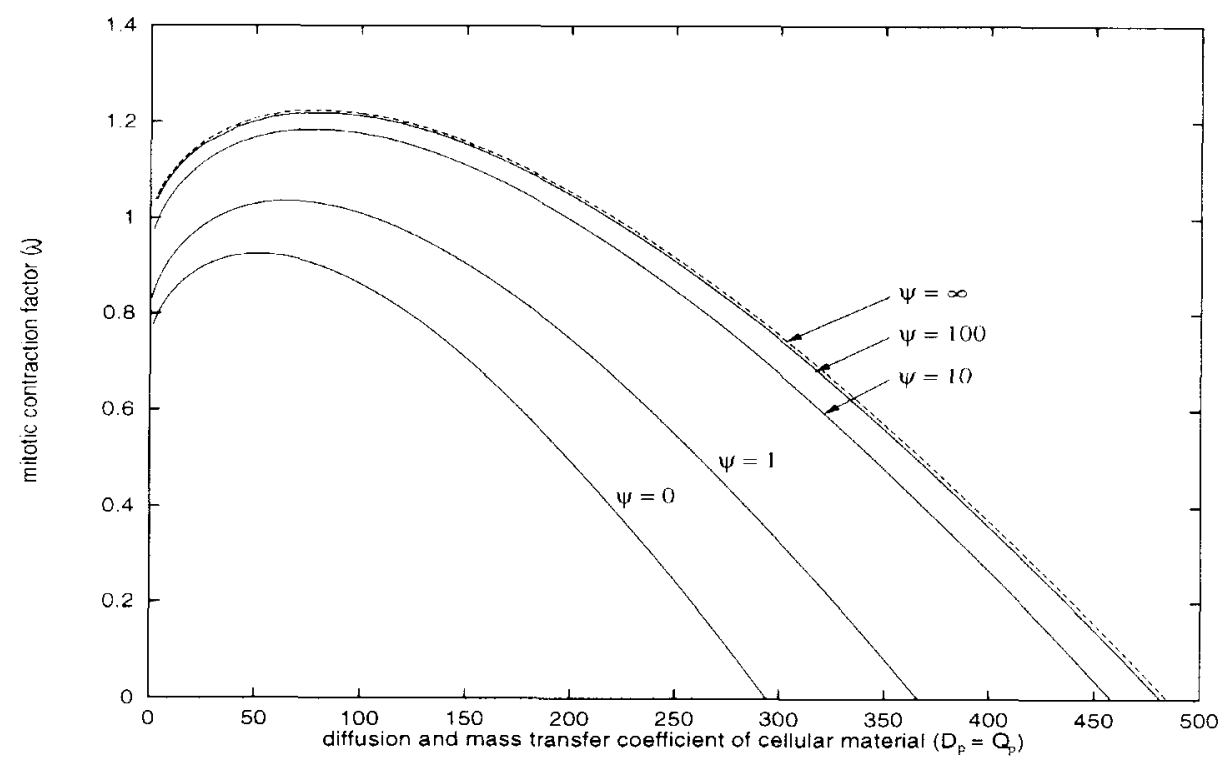

FIGURE 12 The effects of the inhibitor consumption rate $\Psi$ on the travelling wave/steady-state bifurcation in $\left(D_{p}=Q_{p}, \lambda\right)$ space. The dashed curve is the solution of the Inhibitor-free Model with parameters given by (19) and (48).

the nature of the curve changes somewhat, without significantly reducing the area covered by travelling wave solutions. The increased rate of diffusion has meant that the inhibitor escapes from the spheroid so quickly that its effects on the mitotic cells in the viable rim are small, and the change in the curve is due to the mechanism for saturation shifting from the combined inhibition-leakage process to a leakage dominated process. We again note that the system reduces to the Inhibitor-free Model in the limit $D_{h}=Q_{h} \rightarrow \infty$, with a modified value of the cell death contraction factor $\tilde{\delta}=\delta-\mu$. 
Figure 12 shows that increasing the inhibitor consumption rate gives the expected result of expanding the travelling wave region, and the bifurcation curves appear to converge monotonically towards the $\psi \rightarrow \infty$ limit. This limit implies that there is immediate conversion of inhibitor to cellular material by the live cells, and it can show that $h$ tends to zero according to

$$
h \sim \frac{\mu k_{d}(c)}{\psi}
$$

as $\psi \rightarrow \infty$. The system also tends to the Inhibitorfree Model in the limit, with the modified cell death contraction factor $\tilde{\delta}$ now given by $\tilde{\delta}=\delta-(1-\nu) \mu$; the $\psi=\infty$ curve is obtained by this means, again being the same curve as the solid curve shown in Figure 12 of Ward and King (1998).

Shown in the final figure, Figure 13 , is the effect of an externally supplied inhibitor, $h_{0}$, on the saturation size of a spheroid in which no inhibitor is released during necrosis. The inhibitor could be viewed as a drug, provided it is applied in sufficient quantities to contribute a non-negligible proportion of the external medium. The parameters used are given by (19) and (20), except that $\mu=0, \psi=0$ (ensuring no inhibitor is released during necrosis or is broken down) and $P=1$ (to maximise inhibitor effectiveness). We note that the point $h_{0}=0$ is for an uninhibited spheroid and corresponds to the case illustrated by the solid curve in Figure 4, where $S_{x} \approx 168$. From Figure 13 we observe the expected response that $S_{\infty}$ decreases on increasing $h_{0}$. The saturation size reduces sharply in the fully necrotic core region $\left(h_{0}<0.0223\right)$, descending more gently in the partially necrotic core region, before becoming zero at $h_{0}=0.4 ; h_{0}^{*}$, the value of $h_{0}$ at which $S_{\infty} \rightarrow 0$, is given by $k_{m}\left(1, p_{0}, h_{0}^{*}\right)=k_{d}(1)$ (cf. the analysis of Section 5.3). For $h_{0}>h_{0}^{*}$ there are no non-trivial long time solutions, there being sufficiently large concentration of the inhibitors that the death rate always exceeds the birth rate, leading to spheroid extinction. We note that this extinction is due not to the inhibitor killing the tumour cells directly, as would be the case with chemotherapeutic drugs, but through restricting mitosis to a level at which cells die faster than they reproduce.

\section{DISCUSSION}

In this paper we have extended the model of Ward and King (1998) to include a second species of

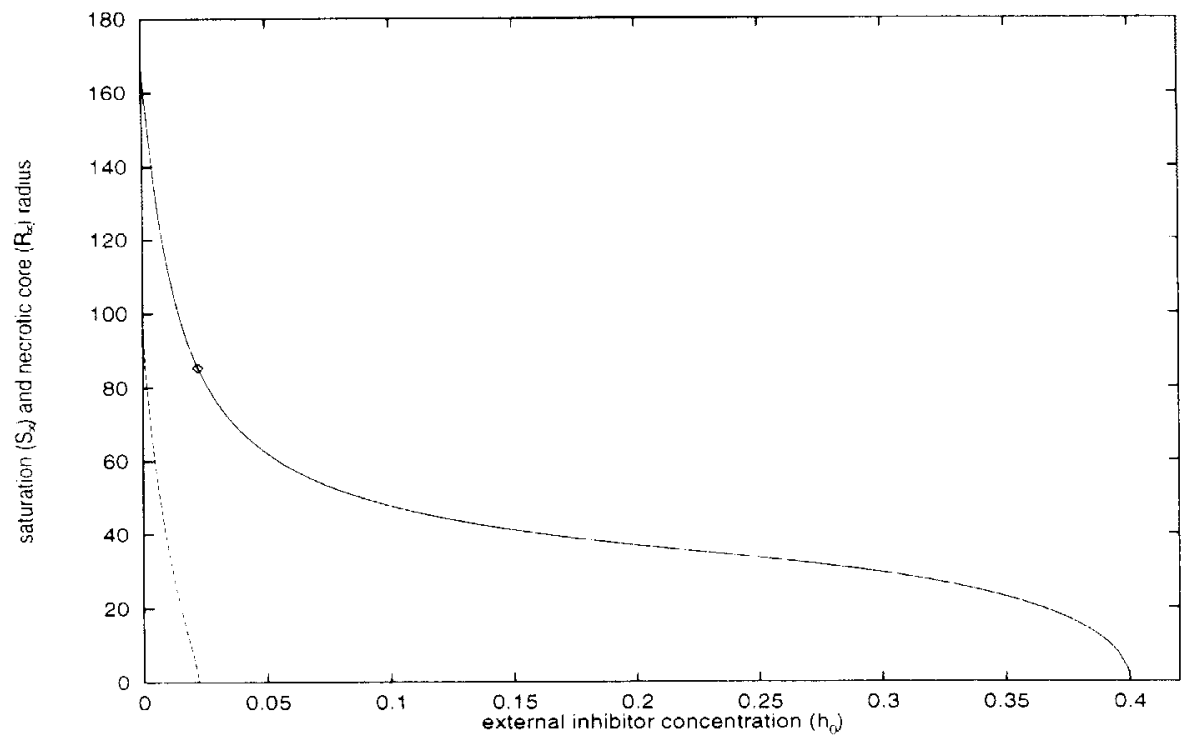

FIGURE 13 The effect of external inhibitor concentration on the saturated spheroid size (solid curve) and necrotic core radius (dashed curve; fully necrotic core interface, $R_{\infty}$ ). The fully/partially necrotic corc bifurcation is indicated by the $\otimes^{\circ}$. 
material released at cell death that has inhibitive properties. The existence of such materials has been demonstrated in numerous experimental studies and it is very likely that their contribution to spheroid growth is significant. The key conclusions are that current of the features of the simpler model of Ward and King (1998), but that the inclusion of the inhibitor can significantly affect the quantitative results. The main difference between the assumptions of the current model and previous models is the manner in which the inhibitor is produced and its diffusion rate. In previous models (Greenspan, 1972; Maggelakis and Adam, 1990) the inhibitor is continually being produced in the necrotic core with no restriction on the concentration it can attain. This is in contrast to our model where there are volumetric restrictions ( $h \leq 1$ in dimensionless terms) and the inhibitor is released only through cell death. However, a model very similar to the previous models can be derived as a limit case of the current one by taking $D_{h}, Q_{h} \rightarrow \infty$, together with $h=\tilde{h} / D_{h}$, $h_{c}=\tilde{h}_{c} / D_{h}, P=1$ and $m_{4} \rightarrow \infty$. These assumptions reduce Equation (16) to a quasi-steady elliptic equation with a step function form for the inhibitor action on the mitotic rate function (10). However, the inhibitive protein molecules at issue are large (molecular mass of $O(10000)$ ) with significantly smaller diffusion rates compared to the nutrients, so that the $D_{h}, Q_{h} \rightarrow \infty$ limit may not be physically realistic. The current model therefore generalises many of the features of previous models and is sufficiently flexible to account for inhibitor molecules of any given size.

Without imposing any a priori assumptions on spheroid structure, the model successfully predicts the exponential, linear and, in appropriate parameter regimes, growth saturation phases together with the observed heterogeneity (necrotic core, quiescent layer and viable rim). The analysis of Sections 5.1 and 5.2 demonstrated that action of the inhibitors alone cannot, except for very a specific set of parameters (namely $\delta-(1-v) \mu=\lambda$ ), force growth saturation; this requires the passage of the either the cellular or the inhibitive material, or both, across the spheroid surface. The numerical solutions show that, even at low concentrations, the inhibitors can have a significant quantitative effect; see for example Figure 4. Figure 4 also demonstrates the two ways in which the 'inhibitor' acts: 1) directly, by reducing the mitotic rate and 2) indirectly, by occupying space, thus reducing the availability of the cellular material. Process 2) is illustrated by the reduction in saturation size for the case of $P=0$ in the figure, while process 1 ) is illustrated by the further reduction in size on increasing $P$. The numerical solutions in Section 6.2 demonstrate that increasing the amount of inhibitor, say by increasing $\mu$ or decreasing $\psi$, leads to the region in which the travelling wave solutions exist being reduced; that is to say, the inhibitor significantly increases propensity for the spheroid to saturate. Although the role of inhibitors may not noticeably effect the behaviour qualitatively, such sensitivity of the solutions to the presence of inhibitors suggests that they play an important complementary role in determining spheroid growth. This indicates that the endogenous production of mitotic inhibitor must be considered in any model intended to give accurate quantitative description of avascular tumour growth.

Our modelling assumes that the inhibitor is produced only from the products of necrosis. It is well-known that cells in normal tissues also produce a number of inhibitory growth factors, and so presumably do cancer cells and cells in vitro. This effect has been considered in the models described in Greenspan (1972) and Maggelakis (1993) and the live cell production of inhibitors can easily be incorporated into the current model. The effects of acidity may also be considered, involving molecules of similar size to the nutrient, and presumably requiring the modelling of the respiratory pathway in a similar manner to Casciari et al. (1992a). These extensions are not expected to change the qualitative behaviour of the current model, but may be significant quantitatively. The passage of necrotic material across the spheroid surface will certainly still be necessary for growth saturation to be predicted.

The extension to the model of Ward and King (1998) has led to a number of new parameters, the values of many of which are unobtainable from data 
currently available. Several studies have isolated individual inhibitor molecules (Harel et al., 1984; Levine et al., 1984), which presumably could be used to provide data from in vitro experimentation. Of these, only Harel et al. (1984) have provided data (namely a value for $P$ ) which could be applied to the current model. Data on the kinetics of mitotic inhibition, necrotic core inhibitor concentration and inhibitor diffusivity would be very useful. Such experiments would have the dual purpose of parameter evaluation and of testing the validity of the modelling assumptions. The current model predicts that the effects of the inhibitor can be significant, even in small quantities (see, for example, Figure 13). Comparison of multicell spheroids grown in a standard medium with those grown in a medium containing an inhibitor would be a relatively simple experiment to confirm, at least qualitatively, this model prediction. Such a substantial response to small concentrations of inhibitor could have implications for the treatment of tumour growth in vivo; the application of drugs that lead to mitotic inhibition, applied in tandem with cell killing drugs, may perhaps lead to more effective anti-cancer therapies.

\section{Acknowledgements}

The first author acknowledges the support of an EPSRC Earmarked Research Studentship and the second a Nuffield Foundation Science Research Fellowship. The authors are also grateful for the referees helpful suggestions for the improvement of this manuscript.

\section{References}

Acker, H., Carlsson, J., Holtermann, G., Nederman, T. and Nylen, T. (1987) Influence of glucose and buffer capacity in the culture medium on growth and $\mathrm{pH}$ in spheroids of human thyroid carcinoma and human glioma origin. Canc. Res., 47, 3504-3508.

Adam, J, A. (1986) A simplified mathematical model of tumour growth. Math. Biosci., 81, 224-229.

Adam, J. A. (1987a) A mathematical model of tumour growth II: Effects of geometry and symmetry. Math. Biosci, 86, $183=211$.

Adam, J. A. (1987b) A mathematical model of tumour growth III: comparison with experiment. Math. Biosci., 86, 213-227.
Adam, J. A. and Maggelakis, S. A. (1989) Mathematical models of tumour growth IV: Effects of necrotic core. Math. Biosci., 97, $121-136$.

Byrne, H. M. and Chaplain, M. A. J. (1996) Growth of necrotic tumours in the presence and absence of inhibitors Math. Biosci., 135, $187-216$.

Carlsson, J. (1997) A proliferation gradient in three-dimensional colonies of cultured human glioma cells. Int. J. Canc., 20, $129-136$.

Casciari, J. J., Sotirchos, S. V. and Sutherland, R. M. (1992a) Mathematical modelling of microenvironment and growth in EMT6/RO multiccllular tumour spheroids. Cell Prolif., 25, $1-22$.

Casciari, J. J., Sotirchos, S. V. and Sutherland, R. M. (1992b) Variation in tumour cell growth rates and metabolism with oxygen-concentration, glucose-concentration and extracellular pH. J. Cell. Physiol., 151, 386-394.

Chaplain, M. A. J. and Britton, N. F. (1993) On the concentration profile of growth-inhibitory factor in multicell spheroids. Math. Biosci., 115, 233-243.

Congar, A. D. and Ziskin, M. C. (1983) Growth of mammalian multicellular tumour spheroids, Canc. Res., 43, 558-560.

Folkman, J. and Hochberg, M. (1973) Self-regulation of growth in three dimensions J. Exp. Med., 138, 745-753.

Freyer, J. P. (1988) Role of necrosis in regulating the growth saturation of multicell spheroids. Canc. Res., 48, 2432-2439.

Freyer, J. P., Schor, P. L. and Saponara, A. G. (1988) Partial purification of a protein-growth inhibitor from multicell spheroids. Biochem. and Biophys. Res. Comm., 152, 463-468.

Freyer, J. P. and Sutherland, R. M. (1986) Regulation of growth saturation and development of necrosis in EMT6/RO multicellular spheroids glucose and oxygen supply. Canc. Res., 46, $3504-3512$.

Greenspan, H. P. (1972) Models for the growth of a solid tumour by diffusion. Stud. Appl. Math., 51, 317-340.

Grocbe, K. and Mueller-Klieser, W. (1996) On the relation between size of necrosis and diameter of tumor spheroids. Int. J. Radiat. Onc. Biol. Phys., 34, 395-401.

Haji-Karim, M. and Carlsson, J. (1978) Proliferation and viability in cellular spheroids of human origin. Canc. Res., 38 $1457-1464$.

Harel, L., Chatelain, G. and Golde, A. (1984) Density dependent inhibition of growth: inhibitory diffusible factors from 3T3 and Rous sarcoma virus (RSV)-transformed 3 T3 cells. J. Cell. Phys., 119, $101-106$.

Inch, W. R., McCredie, J. A. and Sutherland, R. M. (1970) Growth of nodular carcinomas in rodents compared with multicell spheroids in tissuc culture. Growth, 34, 271-282.

Iversen, O. H. (1991) The hunt for endogenous growth inhibitory and or tumour supression factors - their role in physiological and pathological growth regulation. Adv, in Canc: Res., 57, $413-4.53$.

Iwata, K. K., Fryling, C. M., Knott, W. B. and Todaro, G. J. (1985) Isolation of tumour cell growth-inhibiting factors from a human rhabdomysarcoma cell line. Canc: Res., $\mathbf{4 5}$, 2689-2694.

Landry, J., Freyer, J. P and Sutherland, R. M. (1981) Shedding of mitotic cells from the surface of multicell spheroids during growth J. Cell Physiol., 106, 23-32.

Levine, A. E., Hamilton, D. A., Yeoman, L. C. Busch, H. and Brattain, M. G. (1984) Identification of a tumour inhibitory factor in rat ascites fluid. Biochem. Biophys. Res. Comm., 119, $76-82$.

Maggelakis, S. A. and Adam, J. A. (1990) Mathematical model of prevascular growth of a spheroid carcinoma. Math Comput. Mod, , 13, 23-38. 
Maggelakis, S. A. (1993) Mathematical model of prevascular growth of a spheroid carcinoma - part II. Math. Comput. Mod. 17, 19-29.

Nugent, L. and Jain. R. K. (1984) Extravascular diffusion in normal and neoplastic tissues. Canc. Res., 44, 238-244.

Sharma. C. P. and Gehring, H. (1986) A low molecular weight growth inhibitor secreted in cultures of chicken embryo fibroblasts. Biochem. and Biophys. Res. Comm., 139, 1243-1249.

Shymko, R. M. and Glass, L. (1976) Cellular and geometric control of tissue growth and mitotic instability. J. Theo. Biol, 63, $355-374$.

Tannock. I. F. and Kopelyan, I. (1986) Influence of glucoseconcentration on growth and formation of necrosis in spheroids derived from a human bladder cancer cell-line. Canc: Res., 46 , $3105-3110$.
Vaupel, P. W., Frinak, S. and Bicher, H. I. (1981) Heterogenous oxygen partial pressure and $\mathrm{pH}$ distribution in $\mathrm{C} 3 \mathrm{H}$ mouse mammary adenocarcinoma. Can. Res., 41, 2008-2013.

Ward, J. P. (1997) Mathematical modelling of avascular tumour growth. PhD Thesis, Nottingham University.

Ward, J. P. and King, J. R. (1997) Mathematical modelling of avascular-tumour growth. IMA J. Math. Appl. Med., 14, 39-69.

Ward, J. P. and King, J. R. (1998) Mathematical modelling of avascular tumour growth II: modelling growth saturation. IMA J. Math. Appl. Med., [in press].

Weiss, L. (1978) Some mechanisms involved in cancer cell detachment by necrotic material. Int. J. Canc, 22, 196-203. 


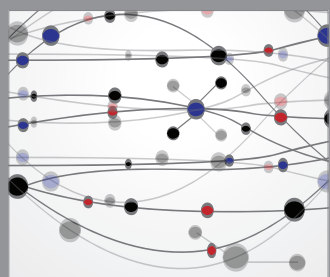

The Scientific World Journal
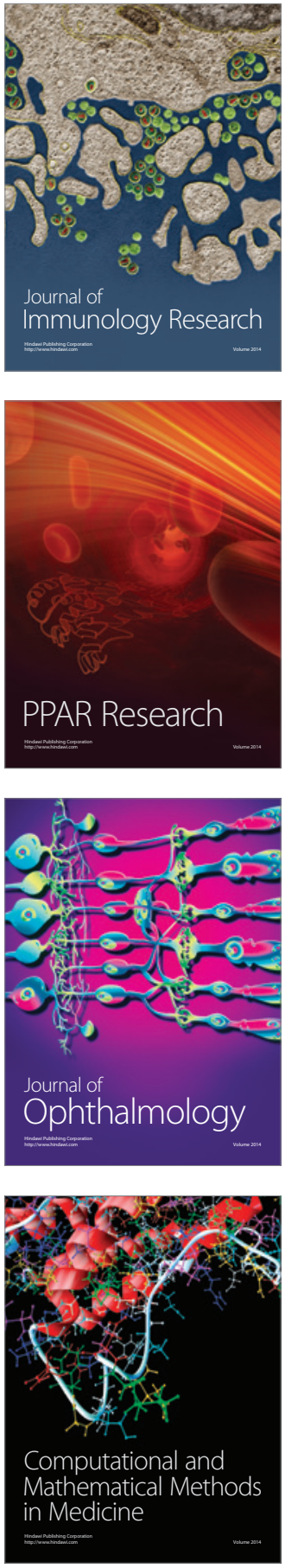

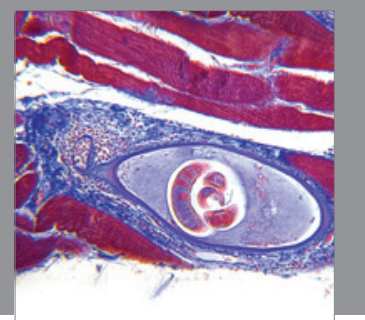

Gastroenterology

Research and Practice
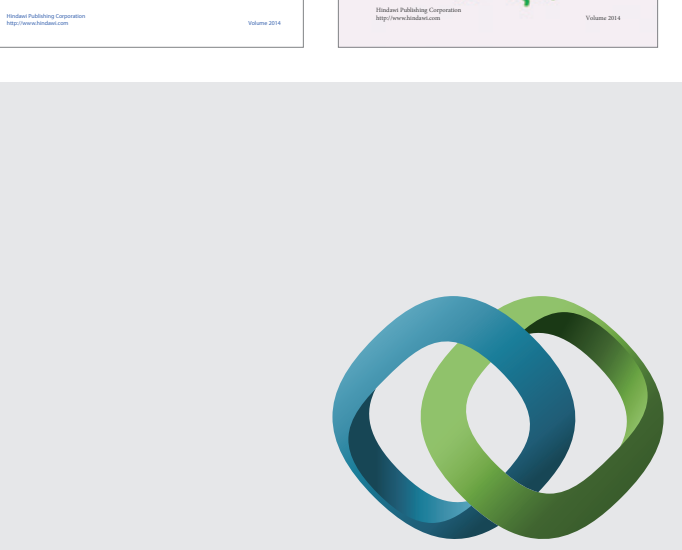

\section{Hindawi}

Submit your manuscripts at

http://www.hindawi.com
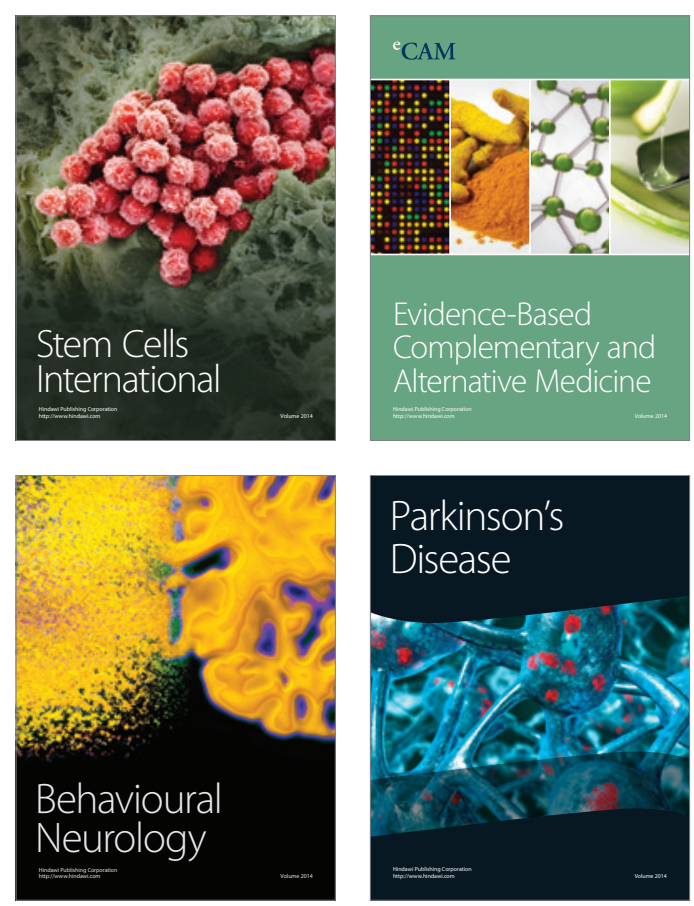

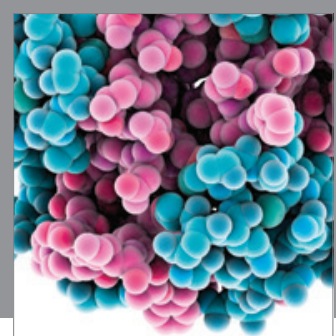

Journal of
Diabetes Research

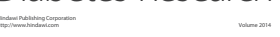

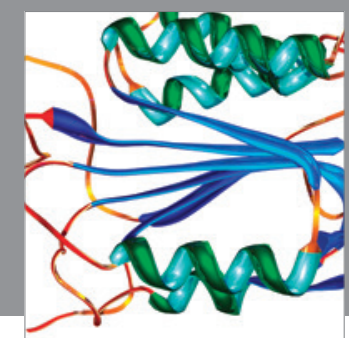

Disease Markers
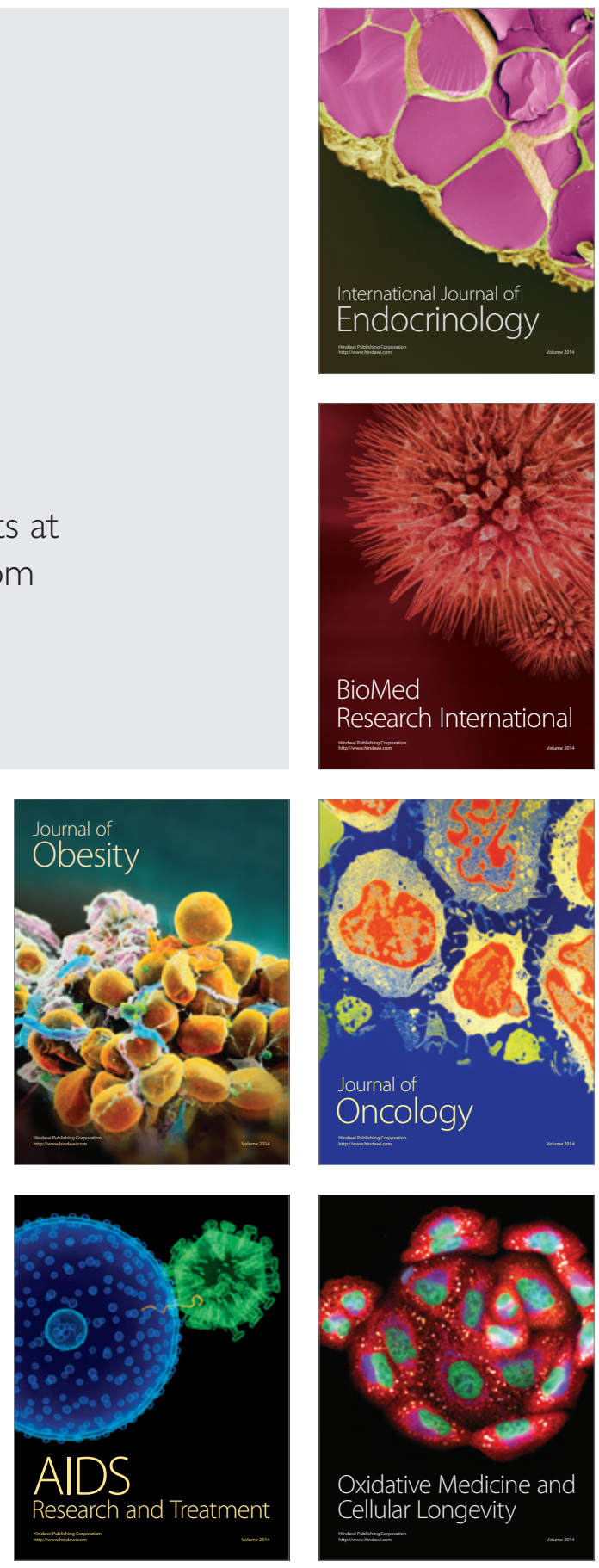